\title{
A Bounded Model Checking Technique for Higher-Order Programs
}

\author{
Yu-Yang Lin and Nikos Tzevelekos \\ Queen Mary University of London
}

\begin{abstract}
We present a Bounded Model Checking technique for higherorder programs based on defunctionalization and points-to analysis. The vehicle of our study is a higher-order calculus with general references. Our technique is a symbolic state syntactical translation based on SMT solvers, adapted to a setting where the values passed and stored during computation can be functions of arbitrary order. We prove that our algorithm is sound and provide a prototype implementation with experimental results showcasing its performance. The first novelty of our technique is a presentation of defunctionalization using nominal techniques, which provides a theoretical background to proving soundness of our technique, coupled with SSA adapted to higher-order values. The second novelty is our use of defunctionalization and points-to analysis to directly encode general higher-order functional programs.
\end{abstract}

\section{Introduction}

Bounded Model Checking [3] (BMC) is a model checking technique that allows for highly automated and scalable SAT/SMT-based verification and has been widely used to find errors in C-like languages 5/15/81. BMC amounts to bounding the executions of programs by unfolding loops only up to a given bound, and model checking the resulting execution graph. Since the advent of CBмс [5], the mainstream approach additionally proceeds by symbolically executing program paths and gathering the resulting path conditions in propositional formulas which can then be passed on to SAT/SMT solvers. Thus, BMC performs a syntactic translation of program source code into a propositional formula, and uses the power of SAT/SMT solvers to check the bounded behaviour of programs. Being a Model Checking technique, BMC has the ability to produce counterexamples, which are execution traces that lead to the violation of desired properties. A specific advantage of BMC over unbounded techniques is that it avoids the full effect of state-space explosion at the expense of full verification. On the other hand, since BMC is inconclusive if the formula is unsatisfiable, it is generally regarded as a bug-finding or underapproximation technique.

The above approach has been predominantly applied to imperative, firstorder languages and, while tools like CBMC can handle C++ (and, more recently, JAVA bytecode), the treatment of higher-order programs is currently limited. In particular, there is no direct analogue of the syntactic translations available for imperative languages in the higher-order case. This is what we address herein. We 
propose a symbolic BMC procedure for higher-order functional and imperative programs that may contain free variables of ground type based on defunctionalization [20] and points-to analysis [2]. Our contributions include: (a) a novel syntactical translation to apply BMC to languages with higher-order methods and state; (b) a proof that the approach is sound; (c) an optimisation based on points-to analysis to improve scalability; (d) and a prototype implementation of the procedure with experimental results showcasing its performance.

As with most approaches to software BMC, we translate a given program into a propositional formula for an SMT solver to check for satisfiability, where formulas are satisfiable only if a violation is reachable within a given bound. Where in first-order programs BMC places a bound on loop unfolding, in the higher-order setting we place the bound on nested method applications. The main challenge for the translation then is the symbolic execution of paths which involve the flow of higher-order terms, by either variable binding or use of the store. We first solve the problem of higher-order store by adapting the standard technique of Static Single Assignment (SSA) to a setting where variables and references can be of higher-order. To handle higher-order terms in particular, we use an approach from operational semantics, whereby each method is uniquely identified by a name. Here, defunctionalization occurs at the semantics level, with methods being passed and stored as unique values, called names, during execution. This acts as a form of defunctionalization by applying each method through a repository. The following section will describe this in more detail. We capture program behaviour by also uniquely identifying every sub-term in the program tree with a return variable; analogous to how CBMC [5] captures the behaviour of sequencing commands in ANSI-C programs.

To give a simple example of our approach, consider the following code, where $r$ is a reference of type int $\rightarrow$ int, $f$ is a variable bound to a method of type int $\rightarrow$ (int $\rightarrow$ int $) \rightarrow$ (int $\rightarrow$ int $) \rightarrow$ (int $\rightarrow$ int), $g, h$ are variables of type int $\rightarrow$ int, and $n, x$ are variables of type int.

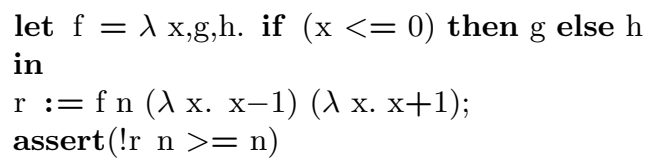

In the code above, a function is assigned to reference $r$. In a symbolic setting, it is not immediately obvious which function to call when dereferencing $r$ in line 4 . Luckily, we know that when calling $f$ in line 3 , its value can only be the one bound to it in line 1. Thus, a first transformation of the code could be:

$3 \mathrm{r}:=$ if $(\mathrm{n}<=0)$ then $(\lambda \mathrm{x} . \mathrm{x}-1)$ else $(\lambda \mathrm{x} . \mathrm{x}+1)$;

$4 \quad \operatorname{assert}($ ! $\mathrm{n}>=\mathrm{n})$

The assignment in line 3 can be facilitated by using a return variable ret and method names for $(\lambda x \cdot x-1)$ and $(\lambda x \cdot x+1)$ :

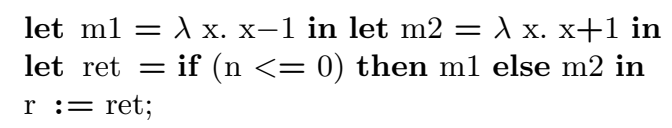


$4 \quad \operatorname{assert}(\ln \mathrm{n}>=\mathrm{n})$

Here lies the challenge as we now need to decide how to symbolically dereference $r$. The simplest solution is try to match $! r$ with all existing functions of matching type, in this case $m 1$ and $m 2$ :

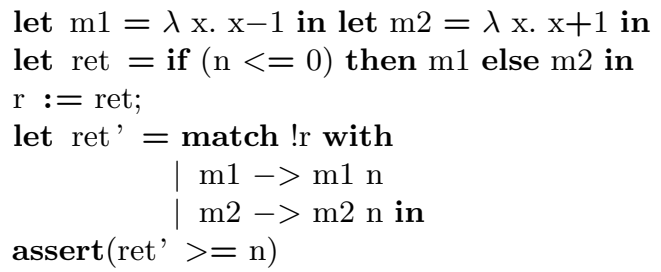

Performing the substitutions of $m 1, m 2$, we can read off the following formula for checking falsity of the assertion:

$$
\begin{aligned}
\left(\text { ret }^{\prime}<n\right) & \wedge\left(r=m 1 \Rightarrow \text { ret }^{\prime}=n-1\right) \wedge\left(r=m 2 \Rightarrow \text { ret }^{\prime}=n+1\right) \wedge(r=\text { ret }) \\
& \wedge(n<=0 \Rightarrow \text { ret }=m 1) \wedge(n>0 \Rightarrow \text { ret }=m 2)
\end{aligned}
$$

The above is true e.g. for $n=0$, and hence the code violates the assertion.

These ideas underpin the core of our BMC translation, which is presented in Section 3 and proven sound later on. The language we examine, HOREF, is a higher-order language with general references and integer arithmetic. While correct, one can quickly see that the translation is inefficient when trying to resolve the flow of functions to references and variables. In effect, it explores all possible methods of the appropriate type that have been created so far, and relies on the solver to pick the right one. This is why a data-flow analysis is required, which we present in Section 4. We optimise the translation by restricting such choices according to a simple points-to analysis. In Section 5 we present an implementation of our technique in a BMC tool for a higher-order OCAML-like syntax extending HOREF, and test it on several example programs adapted from the MoCHi benchmark 13. Finally, we compare our tool with MoCHi and an implementation of our bounded operational semantics in RosETTE [24].

\section{Related Work}

While common in symbolic evaluation, defunctionalization with points-to analysis, to our knowledge, has not been used to translate entire general higher-order programs into SAT/SMT-based BMC encodings. As such, we believe we present a different and sound approach to model checking higher-order terms. BMC being a common technique, there exist several similar encodings. For example, 1076] are bounded approaches based on relational logic that verify JAVA programs using SAT/SMT solvers. Being applied to JAVA, and especially prior to JDK 8, these approaches do not cope with terms and store of arbitrary order. In every case, methods are inlined statically, which is not always possible with function abstractions. In [6] we observe a case of exhaustive method application that restricts concrete method invocations by their type. This is similar in concept to our approach, but is only applied to resolve dynamic dispatch. 
More common are verification tools for general higher-order programs that are not based on a direct syntactical BMC encodings. Two main techniques followed are higher-order recursion schemes modelling [12 18], and symbolic execution 21911. In the first category, MoCHi 13. performs full verification of OCAML programs by translating them into higher-order recursion schemes checked with specialised tools. In the second category, Rosette [24, KAPLAN 14, and RUBICON [16] perform symbolic evaluation for RACKET and RUBY respectively by using solver-aided languages for functional and imperative higher-order programs. On the other hand, the tool implemented in [17] performs a contracts-based symbolic execution that allows evaluating symbolically arbitrarily open higherorder programs. From these approaches, we choose MoCHI and RosetTe as representatives for a comparison in Section 5

Finally, tools based on CBMC are inherently similar to our BMC encoding and procedure as we take inspiration from the CBMC translation, and add symbolic defunctionalization to cope with higher-order syntax. Overall, tools based on symbolic execution are able to produce the most extensionally similar implementations, while intentionally, our approach is closer in idea to CBMC with defunctionalization.

\section{The Language: HORef}

Here we present a higher-order language with higher-order global state. The syntax consists of a call-by-value $\lambda$-calculus with global references. Its types are given by the grammar: $\theta::=$ unit $\mid$ int $|\theta \times \theta| \theta \rightarrow \theta$.

We use countably infinite sets Meths, Refs and Vars for methods, global references and variables, ranged over by $m, r$ and $x$ respectively, and variants thereof; while $i$ is for ranging over the integers. Each of these sets is typed, that is, it can be expressed as a disjoint union as follows: Meths $=\biguplus_{\theta, \theta^{\prime}} \operatorname{Meth}_{\theta, \theta^{\prime}}$, Ref $\mathbf{s}=$ $\biguplus_{\theta} \operatorname{Ref} \mathbf{s}_{\theta}, \quad \operatorname{Vars}=\biguplus_{\theta} \operatorname{Vars}_{\theta}$.

The syntax and typing rules are given in Figure 1. We assume a set of arithmetic operators $\oplus$, which we leave unspecified as they do not affect the analysis. Assertions are used for the specification of safety properties to be checked. We extend the syntax with usual constructs: $r++$ is $r:=! r+1$, and $T ; T^{\prime}$ stands for let $\_=T$ in $T^{\prime}$. Booleans are represented by 0 and $i \neq 0$.

As usual, a variable occurrence is free if it is not in the scope of a matching $(\lambda /$ let/letrec)-binder. Terms are considered modulo $\alpha$-equivalence and, in particular, we may assume that no variable occurs both as free and bound in the same term. We call a term closed if it contains no free variables.

Remark 1. By typing variable, reference and method names, we do not need to provide a context in typing judgements, this choice made for simplicity.

The references we use are global: a term can use references from the set Refs but not create them locally or pass them as arguments, and in particular there is no ref type. Adding ML-like local references is orthogonal to our analysis and it does not seem to present inherent difficulties (we would be treating the dynamic creation of references similarly to how we deal with method names). 


$$
\begin{aligned}
& \text { Terms } \ni T::=\operatorname{assert}(T)|x| m|i|()|\langle T, T\rangle| T \oplus T|r:=T| ! r\left|\pi_{1} T\right| \pi_{2} T \mid \lambda x . T \\
& |T T| \text { if } T \text { then } T \text { else } T \mid \text { let } x=T \text { in } T \mid \text { letrec } x=\lambda x . T \text { in } T \\
& \text { Vals } \ni v::=x|m| i|()|\langle v, v\rangle \\
& \text { ECxts } \ni E::=\bullet|\operatorname{assert}(E)| r:=E|E \oplus T| v \oplus E|\langle E, T\rangle|\langle v, E\rangle\left|\pi_{j} E\right| E T \mid v E \\
& \text { | let } x=E \text { in } T \mid \text { if } E \text { then } T \text { else } T \mid(E) \\
& \text { CForms } \ni M::=\operatorname{assert}(v)|v| r:=v|! r| v \oplus v\left|\pi_{1} v\right| \pi_{2} v|x v| m v \mid \lambda x . M \\
& \text { | if } v \text { then } M \text { else } M \mid \text { let } x=M \text { in } M \mid \text { letrec } x=\lambda x . M \text { in } M
\end{aligned}
$$

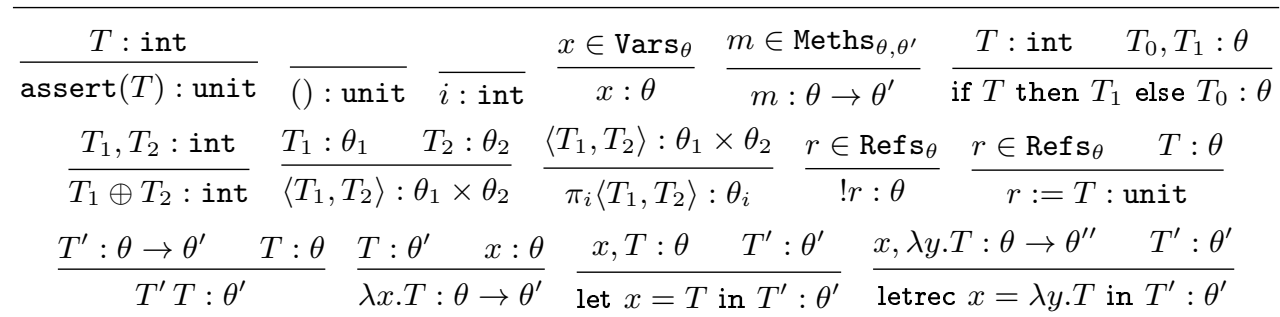

Fig. 1. Grammar and typing rules for HOREF.

On the other hand, methods are dynamically created during execution, and for that reason we will be frequently referring to them simply as names. The terminology comes from nominal techniques [19]. On a related note, $\lambda$-abstractions are not values in our language. This is due to the fact that in the semantics these get evaluated to method names.

Bounded Operational Semantics We next present a bounded operational semantics for HOREF, which we capture with our bounded BMC routine. The semantics is parameterised by a bound $k$ which, similarly to loop unwinding in procedural languages, limits the depth of method (i.e. function) calls within an execution. A bound $k=0$ in particular means that, unless no method calls are made, execution will terminate with no return value. Consequently, in this bounded operational semantics, all programs must halt. Note at this point that the unbounded semantics of HOREF, allowing arbitrary recursion, can be obtained e.g. by allowing bound values $k=\infty$.

The bounded operational semantics is given in Figure 2 It is defined by means of a small-step transition relation with transitions of the form:

$$
(T, R, S, k) \rightarrow\left(T^{\prime}, R^{\prime}, S^{\prime}, k^{\prime}\right)
$$

It uses values and evaluation contexts, which are in turn defined in Figure 1 By abuse of notation, we extended the term syntax to be able to mark nested method calls explicitly, by use of evaluation boxes of the form (...). We use this to correctly bound nested function calls.

A configuration is a quadruple $(T, R, S, k)$ where $T$ is a typed term and: 


$$
\begin{array}{ll}
(\text { assert }(j), R, S, k) \rightarrow((), R, S, k) & (! r, R, S, k) \rightarrow(S(r), R, S, k) \\
(r:=v, R, S, k) \rightarrow((), R, S[r \mapsto v], k) & \left(\pi_{i}\left\langle v_{1}, v_{2}\right\rangle, R, S, k\right) \rightarrow\left(v_{i}, R, S, k\right) \\
\left(i_{1} \oplus i_{2}, R, S, k\right) \rightarrow(i, R, S, k) \quad\left(i=i_{1} \oplus i_{2}\right) & (\lambda x \cdot T, R, S, k) \rightarrow(m, R[m \mapsto \lambda x \cdot T], S, k) \\
\left(\text { if } j \text { then } T_{1} \text { else } T_{0}, R, S, k\right) \rightarrow\left(T_{1}, R, S, k\right) & \left(\text { if } 0 \text { then } T_{1} \text { else } T_{0}, R, S, k\right) \rightarrow\left(T_{0}, R, S, k\right) \\
(\text { let } x=v \text { in } T, R, S, k) \rightarrow(T\{v / x\}, R, S, k) & ((v v), R, S, k) \rightarrow(v, R, S, k+1) \\
(m v, R, S, k) \rightarrow(\mid T\{v / x\}), R, S, k-1) \quad(R(m)=\lambda x \cdot T) \\
\left(\text { letrec } f=\lambda x . T \text { in } T^{\prime}, R, S, k\right) \rightarrow\left(T^{\prime}\{m / f\}, R[m \mapsto \lambda x \cdot T], S, k\right) \\
(E[T], R, S, k) \rightarrow\left(E\left[T^{\prime}\right], R^{\prime}, S^{\prime}, k^{\prime}\right) & \text { where }(T, R, S, k) \rightarrow\left(T^{\prime}, R^{\prime}, S^{\prime}, k^{\prime}\right)
\end{array}
$$

Fig. 2. Bounded operational semantics rules. In all cases, $k \neq \mathrm{nil}$ and $j \neq 0$.

$-R$ : Meths $\rightarrow$ Terms is a finite map, called a method repository, such that for all $m \in \operatorname{dom}(R)$, if $m \in \operatorname{Meths}_{\theta \rightarrow \theta^{\prime}}$ then $R(m)=\lambda x . T: \theta \rightarrow \theta^{\prime}$.

$-S: \operatorname{Refs} \rightarrow$ Vals is a finite map, called a store, such that for all $r \in \operatorname{dom}(S)$, if $r \in \operatorname{Refs}_{\theta}$ then $S(r): \theta$.

$-k \in\{\mathrm{nil}\} \cup \mathbb{N}$ is the nested calling bound, where decrementing $k$ beyond zero results in nil.

A closed configuration is one whose components are all closed. We call a configuration $(T, R, S, k)$ valid if all methods and references appearing in $T, R, S$ are included in $\operatorname{dom}(R)$ and $\operatorname{dom}(S)$ respectively. We also call a transition sequence $(T, R, S, k) \rightarrow\left(T^{\prime}, R^{\prime}, S^{\prime}, k^{\prime}\right)$ valid, where $\rightarrow$ is the reflexive and transitive closure of $\rightarrow$, if $T^{\prime}$ is a value. Note that failing an assertion results to a stuck configuration. Thus, no assertions can be violated in a valid transition sequence. Moreover, we can see that all terms must eventually evaluate to a value, or fail an assertion, or consume the bound and reach nil.

\section{A Bounded Translation for HORef}

We next present an algorithm which, given an initial configuration, produces a tuple containing propositional formulas and context components that capture its bounded semantics. Without loss of generality, we define the translation on terms in canonical form, ranged over by $M$ and variants, which are presented in Figure 1. This provision is innocuous as transforming a term in canonical form can be achieved in linear time with standard methods.

The algorithm receives a valid configuration $(M, R, S, k)$ as input, where $M$ is in canonical form and may only contain free variables of ground type, and proceeds to perform the following sequence of transformations:

$$
(M, R, S, k) \stackrel{i n i t}{\longmapsto}\left(M, R, C_{S}, C_{S}, \phi_{S}, \top, \top, k\right) \stackrel{\llbracket \cdot \rrbracket}{\longmapsto}\left(r e t, \phi, R^{\prime}, C, D, \alpha, p c\right)
$$

The first step is an initialisation step that transforms the tuple in the form appropriate for the main translation $\llbracket \cdot \rrbracket$, which is the essence of the entire bounded translation. We proceed with $\llbracket \cdot \rrbracket$ and will be returning to init later on. 
$\llbracket \cdot \rrbracket$ operates on symbolic configurations of the form $(M, R, C, D, \phi, \alpha, p c, k)$, where $M$ and $R$ are a term and a repository respectively, $k$ is the bound, and:

$-C, D:$ Refs $\rightarrow$ SSAVars are single static assignment (SSA) maps where SSAVars is the set of variables of the form $r_{i}$ (for each $r \in \operatorname{Ref} \mathbf{s}$ ), such that $i$ is the number of times that $r$ has been assigned to so far. The map $C$ is counting all the assignments that have taken place so far, whereas $D$ only counts those in the current path. E.g. $C(r)=r_{5}$ if $r$ has been assigned to five times so far in every path looked at. We write $C[r]$ to mean update $C$ with reference $r$ : if $C(r)=r_{i}$, then $C[r]=C\left[r \mapsto r_{i+1}\right]$, where $r_{i+1}$ is fresh.

$-\phi$ is a propositional formula containing the (total) behaviour so far.

$-\alpha$ is a propositional formula consisting of a conjunction of statements representing assertions that have been visited by $\llbracket \cdot \rrbracket$ so far.

$-p c$ is the path condition that must be satisfied to reach this configuration.

The translation returns tuples of the form ( $r e t, \phi, R, C, D, \alpha, p c)$, where:

- $\phi, R, C, D, \alpha, p c$ have the same reading as above, albeit for after reaching the end of all paths from the term $M$.

- ret is a propositional variable representing the return value of the initial configuration.

Finally, returning to init, we have that:

- the initial SSA maps $C_{S}$ simply map each $r$ in the domain of $S$ to the SSA variable $r_{0}$;

- $\phi_{S}$ stipulates that each $r_{0}$ be equal to its corresponding value $S(r)$;

- since there is no computation preceding $M$, its $\alpha$ and $p c$ are simply $\top$ (true).

The BMC translation is given in Figure 3 In all cases in the figure, ret is a fresh variable and $k \neq$ nil. We also assume a common domain $\Pi=\operatorname{dom}(C)=$ $\operatorname{dom}(D) \subseteq$ Refs, which contains all references that appear in $M$ and $R$.

The translation stops when either the bound is nil, or when every path of the given term has been explored completely. The base cases add clauses mapping return variables to actual values of evaluating $M$. Inductive cases build the symbolic trace of $M$ by recording in $\phi$ all changes to the store, and adding clauses for return variables at each sub-term of the program, thus building a control flow graph by relating said return variables and chaining them together in the formula. Wherever branching occurs, the chaining is guarded.

In the translation, defunctionalization occurs because every method call is replaced with a call to the repository using its respective name as an argument. Because this is a symbolic setting, however, it is possible to lose track of the specific name desired. Particularly, when applying variables as methods $(x v$, with $x: \theta$ ), we encode in the behaviour formula an $n$-ary decision tree where $n$ is the number of methods to consider. In such cases, we assume that $x$ could be any method in the repository $R$. We call this case exhaustive method application. This case seems to be fundamental for applying BMC to higher-order terms with 
state, and is necessary for defunctionalization. To explore plausible paths only, we restrict $R$ to type $\theta$ (denoted $R\lceil\theta$ ). In Section 4) we will be applying a points-to analysis to restrict this further.

To illustrate the algorithm, we look at a few characteristic cases:

[nil case] When the translation consumes its bound, we end up translating some $\llbracket M, R, C, D, \phi, \mathrm{nil} \rrbracket$. In this case, we simply return a fresh variable ret representing the final value, and stipulate in the program behaviour that ret is equal to some default value (the latter is needed to ensure a unique model for ret). The breaching of the bound is recorded as an assertion violation, and a reserved propositional variable inil us used for that purpose. The returned path condition is set to false.

[let case] In \let $x=M$ in $M^{\prime}, R, C, D, \phi, k \rrbracket$, we first compute the translation of $M$. Using the results of said translation, we can substitute in $M^{\prime}$ the fresh variable ret $_{1}$ for $x$, and compute its translation. In the latter step, we also feed the updated repository $R_{1}$, SSA maps $C_{1}$ and $D_{1}$, program behaviour $\phi_{1}$, assertions $\alpha_{1}$ (actually, a conjunction of assertions), and the accumulated path condition $p c \wedge p c_{1}$. To finish, we return $r e t_{2}$ and the newly updated repository $R_{2}$, SSA maps, $C_{2}$ and $D_{2}$, assertions $\alpha_{2}$. The path condition returned is $p c_{1} \wedge p c_{2}$, reflecting the new path conditions gathered.

[ $\mathbf{x v}$ case] In $\llbracket x v, R, C, D, \phi, k \rrbracket$ we see exhaustive method application in action. We first restrict the repository $R$ to type $\theta$ to obtain the set of names identifying all methods of matching type for $x$. If no such methods exist, this means that the binding of $x$ had not succeeded due to breaching the bound earlier on, so defval is returned. Otherwise, for each method $m_{i}$ in this set, we obtain the translation of applying $m_{i}$ to the argument $v$. This is done by substituting $v$ for $y_{i}$ in the body of $m_{i}$. After translating all method applications, all paths are joined in $\psi$, by constructing an $n$-ary decision tree that includes the state of the store in each path. We do this by incrementing all references in $C_{n}$, and adding the clauses $C_{n}^{\prime}=D_{i}(r)$ for each path. These paths are then guarded by the clauses $\left(x=m_{i}\right)$. Finally, we return a formula that includes $\psi$ and the accumulation of all intermediate $\phi_{i}$ 's, the accumulation of repositories, the final SSA map, accumulation of assertions and new path conditions. Note that we return $C_{n}^{\prime}$ as both the $C$ and $D$ resulting from translating this term. This is because all branches have been joined, and any term sequenced after this one should have all updates available to it.

Remark 2. The difference between reading $(D)$ and writing $(C)$ is noticeable when branching. Branching can occur in two ways: through a conditional statement, and by performing symbolic method application where we have lost track of the concrete method; more precisely, when $M$ is of the form $x v$. In the former case, we branch according to the return value of the condition (denoted by ret $_{b}$ ), and each branch translates $M_{0}$ and $M_{1}$ respectively. In this case, both branches read from the same map $D_{b}$, but may contain different assignments, which we accumulate in $C$. The formula $\psi_{0} \wedge \psi_{1}$ then encodes a binary decision node in the control flow graph through guarded clauses that represent the path conditions. Similar care is taken with branching caused by symbolic method application. 


\section{Base Cases:}

$-\llbracket \operatorname{assert}(v), R, C, D, \phi, \alpha, p c, k \rrbracket=($ ret $,($ ret $=()) \wedge \phi, R, C, D,(p c \Longrightarrow(v \neq 0)) \wedge \alpha, \top)$

- $M, R, C, D, \phi, \alpha, p c, \mathrm{nil} \rrbracket=($ ret $,($ ret $=\operatorname{defval}) \wedge \phi, R, C, D, \alpha \wedge(p c \Longrightarrow \operatorname{inil}), \perp)$

- $v v, R, C, D, \phi, \alpha, p c, k \rrbracket=($ ret $,($ ret $=v) \wedge \phi, R, C, D, \alpha, \top)$

- $\llbracket ! r, R, C, D, \phi, \alpha, p c, k \rrbracket=($ ret $,($ ret $=D(r)) \wedge \phi, R, C, D, \alpha, \top)$

- $\lambda \lambda x . M, R, C, D, \phi, \alpha, p c, k \rrbracket=\left(\right.$ ret $,($ ret $\left.=m) \wedge \phi, R^{\prime}, C, D, \alpha, \top\right)$ where $R^{\prime}=R[m \mapsto \lambda x . M]$ and $m$ fresh

- $\llbracket \pi_{i} v, R, C, D, \phi, \alpha, p c, k \rrbracket=\left(\right.$ ret $,\left(\right.$ ret $\left.\left.=\pi_{i} v\right) \wedge \phi, R, C, D, \alpha, \top\right)$

- $v_{1} \oplus v_{2}, R, C, D, \phi, \alpha, p c, k \rrbracket=\left(\right.$ ret $,\left(\right.$ ret $\left.\left.=v_{1} \oplus v_{2}\right) \wedge \phi, R, C, D, \alpha, \top\right)$

- $\llbracket r:=v, R, C, D, \phi, \alpha, p c, k \rrbracket=$ let $C^{\prime}=C[r]$ in let $D^{\prime}=D\left[r \mapsto C^{\prime}(r)\right]$ in

$$
\left(\operatorname{ret},\left((\text { ret }=()) \wedge\left(D^{\prime}(r)=v\right)\right) \wedge \phi, R, C^{\prime}, D^{\prime}, \alpha, \top\right)
$$

\section{Inductive Cases:}

- let $x=M$ in $M^{\prime}, R, C, D, \phi, \alpha, p c, k \rrbracket=$ let $\left(\operatorname{ret}_{1}, \phi_{1}, R_{1}, C_{1}, D_{1}, \alpha_{1}, p c_{1}\right)=\llbracket M, R, C, D, \phi, \alpha, p c, k \rrbracket$ in let $\left(\right.$ ret $\left._{2}, \phi_{2}, R_{2}, C_{2}, D_{2}, \alpha_{2}, p c_{2}\right)=\llbracket M^{\prime}\left\{\operatorname{ret}_{1} / x\right\}, R_{1}, C_{1}, D_{1}, \phi_{1}, \alpha_{1}, p c \wedge p c_{1}, k \rrbracket$ in $\left(\right.$ ret $\left._{2}, \phi_{2}, R_{2}, C_{2}, D_{2}, \alpha_{2}, p c_{1} \wedge p c_{2}\right)$

- letrec $f=\lambda x . M$ in $M^{\prime}, R, C, D, \phi, \alpha, p c, k \rrbracket=$ let $m, f^{\prime}$ be fresh in let $R^{\prime}=R\left[m \mapsto \lambda x . M\left\{f^{\prime} / f\right\}\right]$ in $\llbracket M^{\prime}\left\{f^{\prime} / f\right\}, R^{\prime}, C, D, \phi \wedge\left(f^{\prime}=m\right), \alpha, p c, k \rrbracket$

- $m v, R, C, D, \phi, \alpha, p c, k \rrbracket=$ let $R(m)$ be $\lambda x . N$ in $\llbracket N\{v / x\}, R, C, D, \phi, \alpha, p c, k-1 \rrbracket$

- 【if $v$ then $M_{1}$ else $M_{0}, R, C, D, \phi, \alpha, p c, k \rrbracket=$ let $\left(\right.$ ret $\left._{0}, \phi_{0}, R_{0}, C_{0}, D_{0}, \alpha_{0}, p c_{0}\right)=\llbracket M_{0}, R, C, D, \phi, \alpha, p c \wedge(v=0), k \rrbracket$ in let $\left(\right.$ ret $\left._{1}, \phi_{1}, R_{1}, C_{1}, D_{1}, \alpha_{1}, p c_{1}\right)=\llbracket M_{1}, R_{0}, C_{0}, D_{b}, \phi_{0}, \alpha_{0}, p c \wedge(v \neq 0), k \rrbracket$ in let $C^{\prime}=C_{1}\left[r_{1}\right] \cdots\left[r_{n}\right]\left(\Pi=\left\{r_{1}, \ldots, r_{n}\right\}\right)$ in let $\psi_{0}=(v=0) \Longrightarrow\left(\left(\right.\right.$ ret $=$ ret $\left.\left._{0}\right) \wedge \bigwedge_{r \in \Pi}\left(C^{\prime}(r)=D_{0}(r)\right)\right)$ in let $\psi_{1}=(v \neq 0) \Longrightarrow\left(\left(\right.\right.$ ret $=$ ret $\left.\left._{1}\right) \wedge \bigwedge_{r \in \Pi}\left(C^{\prime}(r)=D_{1}(r)\right)\right)$ in $\left(r e t, \psi_{0} \wedge \psi_{1} \wedge \phi_{1}, R, C^{\prime}, C^{\prime}, \alpha_{1},\left(\left(p c_{0} \wedge(v=0)\right) \vee\left(p c_{1} \wedge(v \neq 0)\right)\right)\right)$

- $x^{\theta} v, R, C, D, \phi, \alpha, p c, k \rrbracket=$ if $R \uparrow \theta=\emptyset$ then $($ ret $,($ ret $=\operatorname{def} v a l) \wedge \phi, R, C, D, \alpha, \perp)$ else let $R \uparrow \theta$ be $\left\{m_{1}, \ldots, m_{n}\right\}$ and $(R, C, \phi, \alpha)$ be $\left(R_{0}, C_{0}, \phi_{0}, \alpha_{0}\right)$ in for each $i \in\{1, \ldots, n\}$ :

let $R\left(m_{i}\right)$ be $\lambda y_{i} . N$ in let $\left(\right.$ ret $\left._{i}, \phi_{i}, R_{i}, C_{i}, D_{i}, \alpha_{i}, p c_{i}\right)=$ $\llbracket N_{i}\left\{v / y_{i}\right\}, R_{i-1}, C_{i-1}, D, \phi_{i-1}, \alpha_{i-1}, p c \wedge\left(x=m_{i}\right), k-1 \rrbracket$ in let $C_{n}^{\prime}=C_{n}\left[r_{1}\right] \cdots\left[r_{j}\right]\left(\Pi=\left\{r_{1}, \ldots, r_{j}\right\}\right)$ in let $\psi=\bigwedge_{i=1}^{n}\left(\left(x=m_{i}\right) \Longrightarrow\left(\left(\right.\right.\right.$ ret $=$ ret $\left.\left.\left._{i}\right) \wedge \bigwedge_{r \in \Pi}\left(C_{n}^{\prime}(r)=D_{i}(r)\right)\right)\right)$ in let $p c_{n}^{\prime}=\bigvee_{i=1}^{n}\left(p c_{i} \wedge\left(x=m_{i}\right)\right)$ in $\left(r e t, \psi \wedge \phi_{n}, R_{n}, C_{n}^{\prime}, C_{n}^{\prime}, \alpha_{n}, p c_{n}^{\prime}\right)$

Fig. 3. The BMC translation. 


$$
\begin{aligned}
& \mathrm{r}:=\mathrm{r} 0 \text {; } \\
& \text { letrec } \mathrm{f}= \\
& \lambda \mathrm{x} \text {. if } \mathrm{x} \text { then }(\mathrm{r}++; \mathrm{f}(\mathrm{x}-1)) \\
& \text { else }(\lambda y \text {. assert }(y=! r+x)) \\
& \text { in let } \mathrm{g}=\mathrm{f} \mathrm{n} \text { in } \mathrm{g} \mathrm{n} \\
& \begin{aligned}
\hline \phi= & \left(r_{1}=r_{0}\right) \wedge\left(\text { ret }_{4}=m_{2}\right) \wedge \\
& \left(\text { ret }_{3}=(n=0) \text { ret }_{4}: \text { ret }_{5}\right) \wedge \\
& \left(r_{2}=r_{1}+1\right) \wedge\left(\text { ret }_{6}=m_{3}\right) \wedge \\
& \left(\text { ret }_{5}=(n-1=0) \text { ret }_{6}: \text { ret }_{7}\right) \wedge \\
& \left(r_{3}=r_{2}+1\right) \wedge\left(\text { ret }_{7}=\operatorname{def}\right) \wedge \\
& \left(\text { ret }_{3}=\operatorname{def} \text { val }\right) \Longrightarrow(\text { ret }=()) \wedge \\
& \left(\text { ret }_{3}=m_{3}\right) \Longrightarrow(\text { ret }=()) \wedge \\
& \left(\text { ret }_{3}=m_{2}\right) \Longrightarrow(\text { ret }=()) \\
\alpha= & \left(\left(\text { ret }_{3}=m_{3}\right) \Longrightarrow\left(n=r_{2}+n-1\right)\right) \wedge \\
& \left(\left(\text { ret }_{3}=m_{2}\right) \Longrightarrow\left(n=r_{1}+n-1\right)\right) \wedge \\
& \left.\left(\left(\text { ret }_{3}=\text { defval }\right) \Longrightarrow \text { inil }\right)\right)
\end{aligned}
\end{aligned}
$$

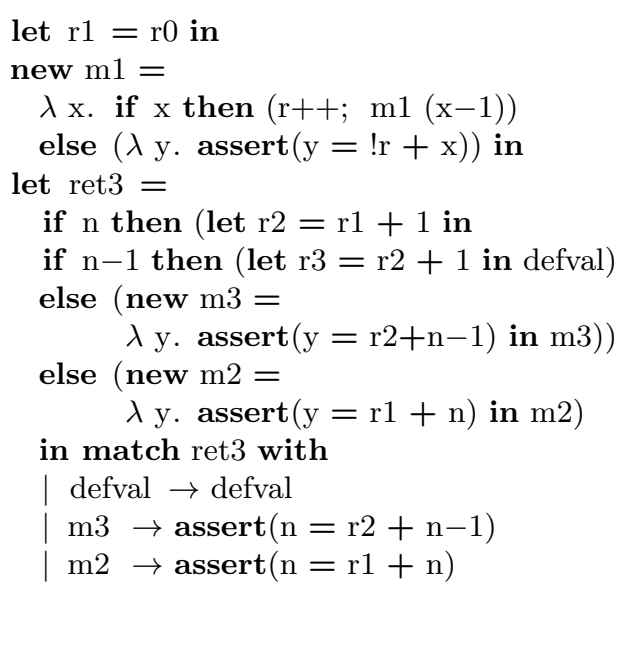

Fig. 4. Translation example (clockwise from top left): original program, unwound program, constraints produced.

Example 3. To illustrate the intuition of our translation, consider the example in Figure 4 for $k=2$. We build a model $\phi$, where, for economy, we directly return variables instead of renaming them, and properties $\alpha$. To construct the formula, we traverse the term in order, and add clauses in order of traversal. Note that the else branch is always explored first. The program is first unwound and transformed to SSA form with exhaustive method application (at match ret3 with). Note that all assignments have been replaced with let-bindings. This is because we convert references to SSA variables. In addition, we use keyword new to add fresh names to the repository.

Bounded Model Checking with the Translation The steps to do a $k$ bounded model checking of some initial configuration using the bounded translation algorithm described previously are as follows. First, given a valid configuration $(M, R, S, k)$, let us set:

$$
C_{S}=\left\{r \mapsto r_{0} \mid r \in \operatorname{dom}(S)\right\} \quad \phi_{S}=\bigwedge_{r \in \operatorname{dom}(S)}\left(r_{0}=S(r)\right)
$$

Thus, starting from $(M, R, S, k)$ :

1. Build $\operatorname{init}(M, R, S, k)=\left(M, R, C_{S}, C_{S}, \phi_{S}, \top, \top, k\right)$.

2. Compute the translation: $\llbracket M, R, C_{S}, C_{S}, \phi_{S}, \top, \top, k \rrbracket=\left(\right.$ ret $\left., \phi, R^{\prime}, C, D, \alpha, p c\right)$.

3. Check $\phi \wedge$ inil $\wedge \neg \alpha$ for satisfiability:

(a) If satisfiable, we have a model for $\phi \wedge$ inil $\wedge \neg \alpha$ that provides values for all open variables $\vec{x}$ and, therefore, a reachable assertion violation.

(b) Otherwise, check $\phi \wedge \neg$ inil $\wedge \neg \alpha$ for satisfiability ${ }^{1}$

\footnotetext{
1 Thus, the role of inil is to switch off/on the recording of reaching the bound as an assertion violation.
} 
i. If satisfiable, the bound was reached, so we increment $k$ by one, and repeat from step (2).

ii. Otherwise, the bound was not reached, so the program has been fully verified.

Soundness In this section we prove that our BMC algorithm is sound for input terms that are closed or contain open variables of ground type.

We start off with some definitions. An assignment $\sigma:$ Vars $\rightarrow$ CVals is a finite map from variables to closed values. Given a term $M$, we write $M\{\sigma\}$ for the term obtained by applying $\sigma$ to $M$. On the other hand, applying $\sigma$ to a method repository $R$, we obtain the repository $R\{\sigma\}=\{m \mapsto R(m)\{\sigma\} \mid m \in \operatorname{dom}(R)\}-$ and similarly for stores $S$. Then, given a valid configuration $(M, R, S, k)$, we have $(M, R, S, k)\{\sigma\}=(M\{\sigma\}, R\{\sigma\}, S\{\sigma\}, k)$.

Given a formula $\psi$ and an assignment $\sigma$, we say $\sigma$ represents $\psi$, and write $\sigma \simeq \psi$, if $\sigma$ satisfies $\psi$ (written $\sigma \vDash \psi$ ) and $\psi$ implies $\sigma(\forall x \in \operatorname{dom}(\sigma) . \psi \Longrightarrow$ $x=\sigma(x)$ ). Given an assignment $\sigma$, we define a formula $\sigma^{\circ}$ representing it by: $\sigma^{\circ}=\bigwedge_{x \in \operatorname{dom}(\sigma)}(x=\sigma(x))$.

We define $\llbracket M, R, S, k \rrbracket=\llbracket \operatorname{init}(M, R, S, k) \rrbracket$.

Theorem 4 (Soundness). Given a valid configuration $(M, R, S, k)$ whose open variables are of ground type, suppose $\llbracket M, R, S, k \rrbracket=\left(\right.$ ret $\left., \phi, R^{\prime \prime}, C, D, \alpha, p c\right)$. Then, for all assignments $\sigma_{0}$ closing $(M, R, S, k), 1$ and 2 are equivalent:

1. $\exists E, R^{\prime}, S^{\prime} .(M, R, S, k)\left\{\sigma_{0}\right\} \rightarrow\left(E[\operatorname{assert}(0)], R^{\prime}, S^{\prime}, k^{\prime}\right)$

2. $\exists \sigma^{\prime} \supseteq \sigma_{0} . \sigma^{\prime} \models \phi \wedge$ inil $\wedge \neg \alpha$.

Moreover, if $\phi \wedge$ inil $\wedge \neg \alpha$ is not satisfiable then 3 and 4 are equivalent:

3. $\exists M^{\prime}, R^{\prime}, S^{\prime} .(M, R, S, k)\left\{\sigma_{0}\right\} \rightarrow\left(M^{\prime}, R^{\prime}, S^{\prime}, \mathrm{nil}\right)$

4. $\exists \sigma^{\prime} \supseteq \sigma_{0} . \sigma^{\prime} \models \phi \wedge \neg$ inil $\wedge \neg \alpha$.

Proof. (1) $\Longrightarrow(2)$ and (3) $\Longrightarrow$ (4) follow directly from Lemma 5 below. For the reverse directions, we rely on the fact that the semantics is bounded so, in every case, $(M, R, S, k)\left\{\sigma_{0}\right\}$ should either reach a value, or a failed assertion, or hit the bound. Moreover, the semantics is deterministic, in the sense that the configurations that can be eventually reached may only differ in the choice of fresh names used in the transition sequence (see Appendix A). These two facts allow us to employ Lemma 5 also for the reverse directions.

Lemma 5 (Correctness). Given $M, R, C, D, \phi, \alpha, p c, k, \sigma$ such that $\sigma \simeq \phi$, $(M, R, D, k)\{\sigma\}$ is valid, and $\llbracket M, R, C, D, \phi, \alpha, p c, k \rrbracket=\left(\right.$ ret $\left., \phi^{\prime}, R^{\prime}, C^{\prime}, D^{\prime}, \alpha^{\prime}, p c^{\prime}\right)$ there exists $\sigma^{\prime} \supseteq \sigma$ such that $\sigma^{\prime} \simeq \phi^{\prime}$ and:

- if $(M, R, D, k)\{\sigma\} \rightarrow(v, \hat{R}, \hat{S}, \hat{k})$ then $\sigma^{\prime} \vDash\left(p c \Longrightarrow\left(p c^{\prime} \wedge(\right.\right.$ ret $\left.\left.=v)\right)\right) \wedge$ $\left((\right.$ inil $\left.\wedge \alpha \wedge p c) \Longrightarrow \alpha^{\prime}\right), R^{\prime}\left\{\sigma^{\prime}\right\} \supseteq \hat{R}$, and $D^{\prime}\left\{\sigma^{\prime}\right\}=\hat{S}$.

- if $(M, R, D, k)\{\sigma\} \rightarrow(E[\operatorname{assert}(0)], \hat{R}, \hat{S}, \hat{k})$ then $\sigma^{\prime} \vDash((\operatorname{inil} \wedge \alpha \wedge p c) \Longrightarrow$ $\left.\neg \alpha^{\prime}\right)$

- if $(M, R, D, k)\{\sigma\} \rightarrow(\hat{M}, \hat{R}, \hat{S}$, nil $)$ then $\sigma^{\prime} \vDash\left(p c \Longrightarrow \neg p c^{\prime}\right) \wedge(($ inil $\wedge \alpha \wedge$ $\left.p c) \Longrightarrow \alpha^{\prime}\right) \wedge\left((\neg\right.$ inil $\left.\wedge \alpha \wedge p c) \Longrightarrow \neg \alpha^{\prime}\right)$. 


\section{Base Cases:}

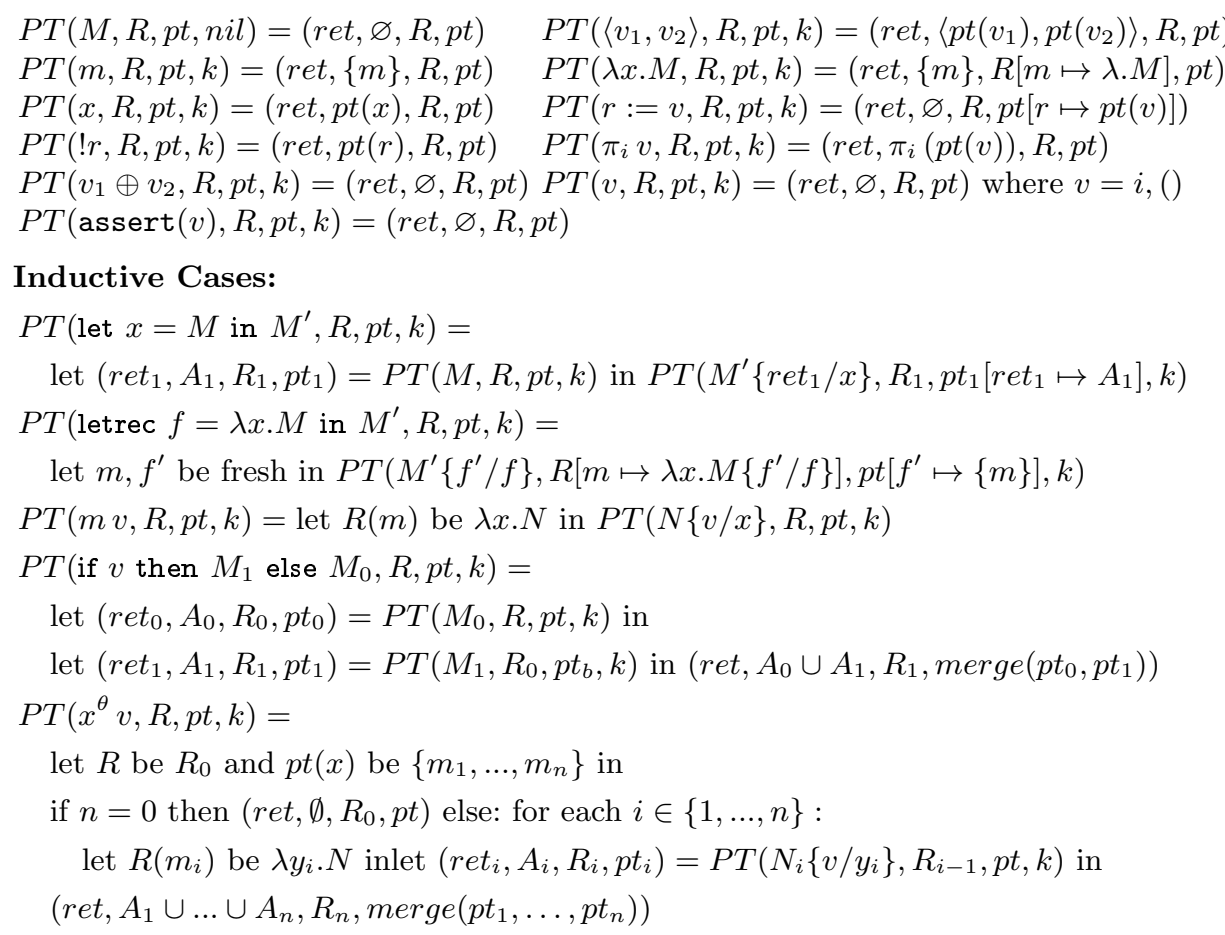

Fig. 5. The points-to analysis algorithm.

\section{A Points-to Analysis for Names}

The presence of exhaustive method application in our BMC translation is a primary source of state space explosion. As such, a more precise filtering of $R$ is necessary for scalability. In this section we describe a simple analysis to restrict the number of methods considered. We follow ideas from points-to analysis, which typically computes an overapproximation of the points-to set of each variable inside a program, that is, the set of locations that it may point to.

Our analysis computes the set of methods that may be bound to each variable while unfolding and. We do this via a finite map $p t:(\operatorname{Ref} \mathbf{s} \cup \operatorname{Vars}) \rightarrow$ Pts where Pts contains all points-to sets and is given by: Pts $\ni A::=X \mid\langle A, A\rangle$ where $X \subseteq_{\text {fin }}$ Meths. Thus, a points-to set is either a finite set of names or a pair of points-to sets. These need to be updated whenever a method name is created, and are assigned to references or variables according to the following cases:

$$
\begin{array}{ll}
r:=M & \text { add in } p t: r \mapsto p t(M) \\
\text { let } x=M \text { in } M^{\prime} & \text { add in } p t: x \mapsto p t(M) \\
x M & \text { add in } p t: \operatorname{ret}(M) \mapsto p t(M)
\end{array}
$$

where $\operatorname{ret}(M)$ is the variable assigned to the result of $M$. The letrec binder follows a similar logic. The need to have sets of names, instead of single names, in 
the range of $p t$ is that the analysis, being symbolic, branches on conditionals and applications, so the method pointed to by a reference cannot be decided during the analysis. Thus, when joining after branching, we merge the $p t$ maps obtained from all branches.

The points-to algorithm is presented in Figure 5 Given a valid configuration $(M, R, S, k)$, the algorithm returns $P T(M, R, S, k)=($ ret $, A, R, p t)$, where $A$ is the points-to set of ret, and $p t$ is the overall points-to map computed.

The merge of points-to maps is given by:

$$
\operatorname{merge}\left(p t_{1}, \ldots, p t_{n}\right)=\left\{x \mapsto \bigcup_{i} \hat{p} t_{i} \mid x \in \bigcup_{i} \operatorname{dom}\left(p t_{i}\right)\right\}
$$

where $\hat{p t}_{i}(x)=p t_{i}(x)$ if $x \in \operatorname{dom}\left(p t_{i}\right), \emptyset$ otherwise, and $A \cup B$ is defined by $\left\langle A_{1} \cup B_{1}, A_{2} \cup B_{2}\right\rangle$ if $A, B=\left\langle A_{1}, A_{2}\right\rangle,\left\langle B_{1}, B_{2}\right\rangle$, and just $A \cup B$ otherwise.

The optimised BMC translation We can now incorporate the points-to analysis in the BMC translation to get an optimised translation which operates on symbolic configurations augmented with a points-to map, and returns:

$$
\llbracket M, R, C, D, \phi, \alpha, p c, p t, k \rrbracket_{P T}=\left(r e t, \phi^{\prime}, R^{\prime}, C^{\prime}, D^{\prime}, \alpha, p c, A, p t^{\prime}\right)
$$

The optimised BMC translation is defined by lock-stepping the two algorithms presented above (i.e. $\llbracket-\rrbracket$ and $P T\left(_{-}\right)$) and letting $\llbracket-\rrbracket$ be informed from $P T\left(_{-}\right)$in the $x M$ case, which now restricts the choices of names for $x$ to the set $p t(x)$. Its soundness is proven along the same lines as the basic algorithm.

To illustrate the significance of reducing the set of names, consider the program on the right which recursively generates names to compute triangular numbers. Without points-to analysis, since $f$ creates a new method per call, and the translation considers all methods of matching type per recursive call, the number of names to apply at depth $m \leq n$ when translating $f(n)$ is approximately $m$ !. This means that the number

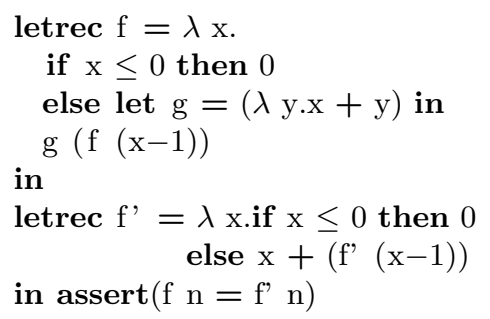

of paths explored grows by the factorial of $n$, with the total number of methods created being the left factorial sum $! n$, and total number of names considered being the derangement of $n$. In contrast, $f^{\prime}(n)$ only considers $n$ names with a linear growth in number of paths. With points-to analysis, the number of names considered and created in $f$ is reduced to that of $f^{\prime}$.

\section{Implementation and Experiments}

We implemented the translation algorithm in a prototype tool to model check higher-order programs called BMC-2. The implementation and benchmarks can be found at: 
The tool takes program source code written in an ML-like language, and produces a propositional formula in SMT-LIB 2 format. This is then be fed to an SMT solver such as Z3. Syntax of the input language is based on the subset of OCAML that corresponds to HOREF. Differences between OCAML and our concrete syntax are for ease of parsing and lack of type checking. For instance, all input programs must be either written in "Barendregt Convention", meaning all bound variables must be fresh, or such that variables have the same type globally. Additionally, all bound variables are annotated with types. Internally, BMC-2 implements an abstract syntax that extends HOREF with vector arguments and integer lists. This means that functions can take multiple arguments at once. Lists are handled for testing, but not discussed here as they are not relevant to the theory. BMC-2 itself was written in OCAML.

To illustrate our input language, on the right is sample program mc91-e from [13] translated from OCAML to our syntax. The keyword Methods is used to define all methods in the repository. The keyword Main is used to define the main method. For this sample program, our tool builds a translation with $k=1$ for which Z3 correctly reports that the assertion fails if $n=102$.

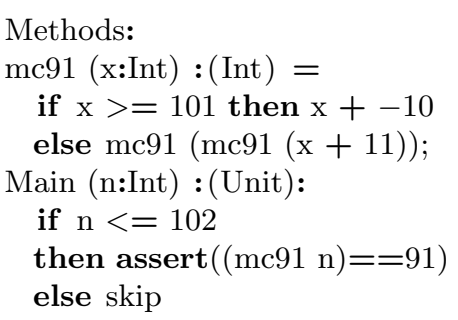
Details about experiments will be provided later.

Benchmarks We tested our implementation on a set of 40 programs that include a selection from the MoCHI benchmark [13; a set of higher-order programs written in OCAML, originally used to test the higher-order model checking tool MoCHI 13, and subsequently used for benchmarking [23/4/22]. We added custom samples with references (ref-1, ref-1-e, ref-2, ref-2-e, ref-3), as well as programs of varying lengths-100, 200 and 400 lines of code-constructed by combining the other samples. To combine programs, we refactored and concatenated methods and main methods from different files into a single file, and switch between the methods based on user input, thus forcing BMC-2 to consider all mains. In this set we have of safe and unsafe programs denoted by the -e termination in their filename. Unsafe programs were constructed by slight modifications to the assertions of the original safe programs. For our experiment, the programs were manually translated to our input language and checked using our tool and Z3. Care was taken to keep all sample programs as close to the original source code as our concrete syntax allows. All experiments ran on an UBUNTU machine equipped with an Intel Core i7-6700 CPU clocked at 3.40GHz and 16GB RAM. All tests were set to time-out after 3 minutes, and up to a maximum bound $k=15$. These limits were chosen due to the combinatorial nature of model checking and the sample programs used. BMC-2 ran twice per program per bound, and the average was recorded.

Figure 6 plots the average time taken for BMC-2 to check all the benchmark programs. We can observe that performance of BMC-2 heavily depends on the program it is checking, making the possibility of full verification entirely dependent on the nature of the program. For example, ack, which is an implementation of 


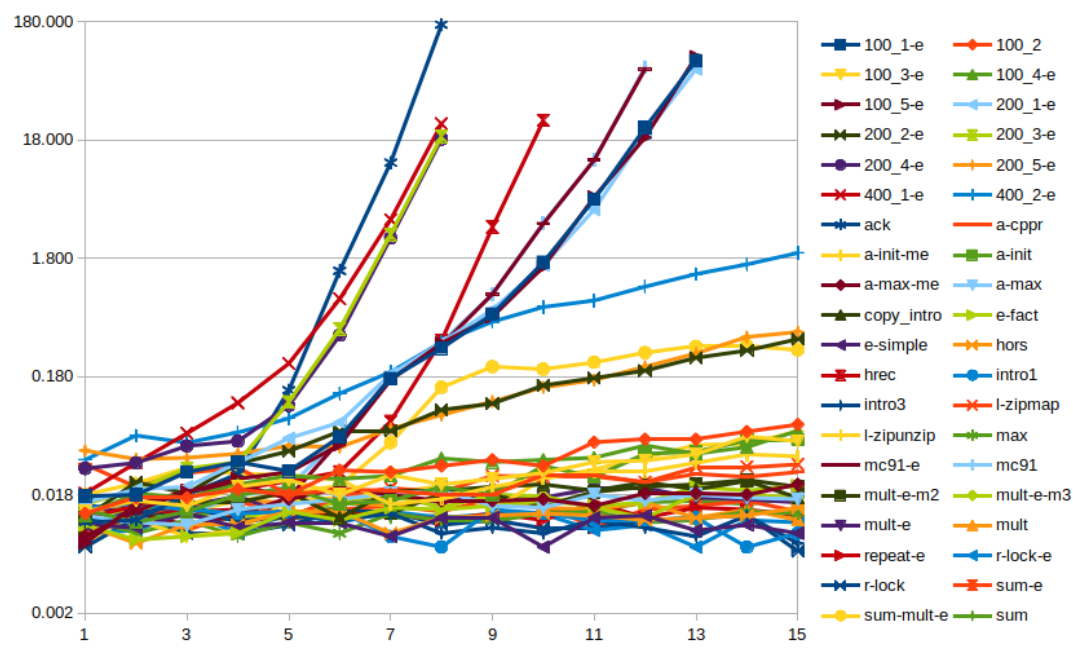

Fig. 6. Average execution time (s) for BMC-2 on bounds $k=1 . .15$.

the Ackermann function, is a deeply recursive program that diverges rapidly, and thus cannot be translated by our algorithm any better than its normal growth. This agrees with the intuition that BMC is not appropriate to find bugs in deep recursion. As mentioned before, however, BMC has been shown to be effective on shallow bugs in industry. This can be seen with our examples for 100 to 400 lines of code, which were correctly shown to have bugs, with little difficulty despite the increase in program size.

In addition to testing $\mathrm{BMC}-2$, we also ran comparison experiments on prior tools MoCHi [13] and Rosette [24. All experiments ran on the same machine used to test BMC-2. These tools will be described in more detail in the following section. For RosetTe, we used an implementation of our bounded semantics in RosetTe provided to us by an anonymous reviewer. With the semantics implemented, we compare RoseTTE's symbolic execution of HOREF, to BMC-2 with Z3's translation and solving of the same terms.

\begin{tabular}{l|c|c|c|c|c|c}
\hline & 4 & 7 & 10 & 13 & 15 & $\mathrm{MoCHi}$ \\
\hline 100_1-e & 0.034 & 0.173 & 1.661 & 84.130 & - & $\mathrm{c}$ \\
100_2 & 0.020 & 0.028 & 0.032 & 0.053 & 0.071 & $\mathrm{c}$ \\
100_3-e & 0.021 & 0.027 & 0.028 & 0.040 & 0.051 & 10.734 \\
200_1-e & 0.034 & 0.188 & 1.572 & 71.296 & - & $\mathrm{m}$ \\
200_2-e & 0.033 & 0.063 & 0.151 & 0.259 & 0.372 & - \\
200_3-e & 0.034 & 2.849 & - & - & $\mathrm{m}$ & 1.742 \\
$400 \_1-e$ & 0.108 & 3.805 & - & - & $\mathrm{m}$ & $\mathrm{m}$ \\
400_2-e & 0.061 & 0.196 & 0.696 & 1.321 & 1.991 & - \\
ack & 0.027 & 11.519 & - & - & - & 0.525 \\
a-cppr & 0.031 & 0.020 & 0.026 & 0.027 & 0.028 & 28.584 \\
a-init & 0.018 & 0.016 & 0.032 & 0.042 & 0.053 & $\mathrm{c}$ \\
e-fact & 0.009 & 0.014 & 0.016 & 0.021 & 0.022 & 0.629 \\
e-simple & 0.010 & 0.008 & 0.007 & 0.009 & 0.009 & 0.098 \\
hrec & 0.020 & 0.075 & 26.175 & - & - & 0.867 \\
r-lock-e & 0.009 & 0.013 & 0.013 & 0.007 & 0.011 & 0.216 \\
ref-2 & 0.013 & 0.008 & 0.011 & 0.012 & 0.010 & $\mathrm{u}$ \\
ref-2-e & 0.011 & 0.013 & 0.010 & 0.013 & 0.011 & $\mathrm{u}$ \\
ref-3 & 0.019 & 0.018 & 0.047 & 0.211 & 0.211 & $\mathrm{u}$ \\
\hline
\end{tabular}

Comparison with MoCHi Though the goals of each tool are different, we attempted to compare our approach to MoCH. Being unable to build from source, Table 1. Time taken (s) for BMC-2 $(k=1 . .15)$ and $\mathrm{MoCHi}$, where,$- c$, $m$ and $u$ respectively indicate timeout, crash, out-of-memory and unsupported we decided to used the Dockerfile on the UBUNTU machine from before. In Table 1 . we have the time taken for BMC-2 and MoCHi for a smaller set of programsthe full range of results can be found in Appendix C. We noticed that MoCHI is very sensitive to the operators and functions used in the assertions, while 
BMC-2 appears to be less dependent on these. For instance, checking mult-e with assert(mult $\mathrm{m} \mathrm{m}<=$ mult $\mathrm{n} \mathrm{n}$ ) was three orders of magnitude slower than the original, while, at $k=1$, BMC-2 takes 0.012 seconds; an increase of $20 \%$ from the original $0.010 \mathrm{~s}$ to find a bug. We also noticed that MoCHI is less consistent with larger programs. For 100 to 400 lines of code, MoCHI correctly found bugs in 4 out of 12 samples, but halted unexpectedly on the remaining 8 . BMC-2 found all 11 bugs of the 12 programs, and found no bugs in the safe program. Finally, we included 5 examples with references, which BMC-2 correctly checked. Attempting to check them with MoCHI suggests references are unsupported.

Comparison with Rosette for Racket We found that BMC-2 and RoseTTE are very similar in their ability to check higher-order programs. Since RACKET is a stateful higher-order language like HOREF, and RosETTE employs a symbolic virtual machine with symbolic execution techniques for RACKET, we can expect this similarity. Fundamentally, RosetTe and BMC-2 provide different approaches to verification as the former is related to symbolic evaluation, while the latter is a monolithic BMC translation. We were particularly interested in RosETTE's ability to implement bounded verification for higher-order programs. We defined our bounding mechanism in RoseTTE, and compared its symbolic evaluation to BMC-2 on the UBUNTU machine. Figure 7 showcases this comparison. We found

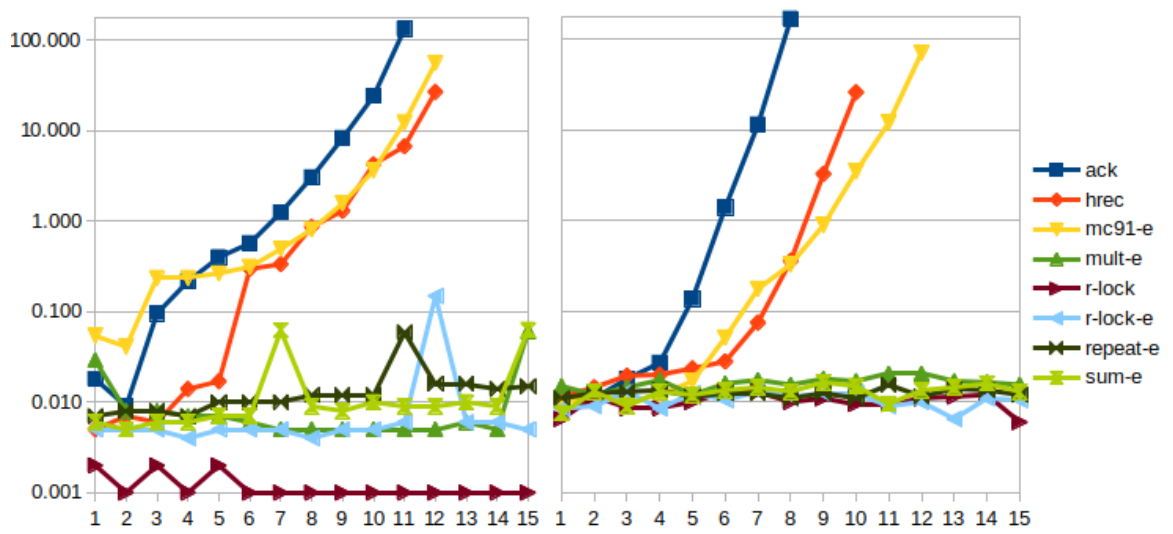

Fig. 7. Execution time (s) of $k=1 \ldots 15$ for Rosette (left) and BMC-2 (right).

that RosetTe and BMC-2 are comparable in scalability, with BMC-2 being less optimised for some diverging programs such as ack. This could be due to the way ROSETTE performs type-driven state merging, which provides opportunities for concretization. In contrast, we perform a suboptimal SSA transformation which could benefit from dominance frontiers for optimal merging of control flow. BMC, however, has the theoretical advantage of faster compilation time over symbolic execution 24. Since testing in RoseTte involves manually (re)translating our examples into RACKET, our comparison is based on a sample of 8 programs from the MoCHI benchmarks. 


\section{References}

1. N. Amla, R. P. Kurshan, K. L. McMillan, and R. Medel. Experimental analysis of different techniques for bounded model checking. In H. Garavel and J. Hatcliff, editors, Tools and Algorithms for the Construction and Analysis of Systems, 9th International Conference, TACAS 2003, Proceedings, volume 2619 of Lecture Notes in Computer Science, pages 34-48. Springer, 2003.

2. L. O. Andersen. Program Analysis and Specialization for the C Programming Language. PhD thesis, DIKU, University of Copenhagen, May 1994. (DIKU report 94/19).

3. A. Biere, A. Cimatti, E. M. Clarke, and Y. Zhu. Symbolic model checking without bdds. In R. Cleaveland, editor, Tools and Algorithms for Construction and Analysis of Systems, 5th International Conference, TACAS '99, Proceedings, volume 1579 of Lecture Notes in Computer Science, pages 193-207. Springer, 1999.

4. T. C. Burn, C. L. Ong, and S. J. Ramsay. Higher-order constrained horn clauses for verification. PACMPL, 2(POPL):11:1-11:28, 2018.

5. E. M. Clarke, D. Kroening, and F. Lerda. A tool for checking ANSI-C programs. In K. Jensen and A. Podelski, editors, Tools and Algorithms for the Construction and Analysis of Systems, 10th International Conference, TACAS 2004, Proceedings, volume 2988 of Lecture Notes in Computer Science, pages 168-176. Springer, 2004.

6. G. Dennis, F. S. Chang, and D. Jackson. Modular verification of code with SAT. In L. L. Pollock and M. Pezzè, editors, Proceedings of the ACM/SIGSOFT International Symposium on Software Testing and Analysis, ISSTA 2006, Portland, Maine, USA, July 17-20, 2006, pages 109-120. ACM, 2006.

7. J. Dolby, M. Vaziri, and F. Tip. Finding bugs efficiently with a SAT solver. In I. Crnkovic and A. Bertolino, editors, Proceedings of the 6th joint meeting of the European Software Engineering Conference and the ACM SIGSOFT International Symposium on Foundations of Software Engineering, 2007, Dubrovnik, Croatia, September 3-7, 2007, pages 195-204. ACM, 2007.

8. V. D'Silva, D. Kroening, and G. Weissenbacher. A survey of automated techniques for formal software verification. IEEE Trans. on CAD of Integrated Circuits and Systems, 27(7):1165-1178, 2008.

9. W. E. Howden. Symbolic testing and the dissect symbolic evaluation system. Software Engineering, IEEE Transactions on, SE-3:266- 278, 081977.

10. J. P. Galeotti, N. Rosner, C. G. L. Pombo, and M. F. Frias. TACO: efficient sat-based bounded verification using symmetry breaking and tight bounds. IEEE Trans. Software Eng., 39(9):1283-1307, 2013.

11. J. King. A new approach to program testing. 10:228-233, 061975.

12. N. Kobayashi. Types and higher-order recursion schemes for verification of higherorder programs. In Proceedings of the 36th Annual ACM SIGPLAN-SIGACT Symposium on Principles of Programming Languages, POPL '09, pages 416-428, New York, NY, USA, 2009. ACM.

13. N. Kobayashi, R. Sato, and H. Unno. Predicate abstraction and CEGAR for higher-order model checking. In M. W. Hall and D. A. Padua, editors, Proceedings of the 32nd ACM SIGPLAN Conference on Programming Language Design and Implementation, PLDI 2011, pages 222-233. ACM, 2011.

14. A. S. Köksal, V. Kuncak, and P. Suter. Constraints as control. In J. Field and M. Hicks, editors, Proceedings of the 39th ACM SIGPLAN-SIGACT Symposium on Principles of Programming Languages, POPL 2012, Philadelphia, Pennsylvania, USA, January 22-28, 2012, pages 151-164. ACM, 2012. 
15. J. Morse, M. Ramalho, L. C. Cordeiro, D. Nicole, and B. Fischer. ESBMC 1.22 - (competition contribution). In E. Ábrahám and K. Havelund, editors, Tools and Algorithms for the Construction and Analysis of Systems - 20th International Conference, TACAS 2014, Proceedings, volume 8413 of Lecture Notes in Computer Science, pages 405-407. Springer, 2014.

16. J. P. Near and D. Jackson. Rubicon: bounded verification of web applications. In W. Tracz, M. P. Robillard, and T. Bultan, editors, 20th ACM SIGSOFT Symposium on the Foundations of Software Engineering (FSE-20), SIGSOFT/FSE'12, Cary, NC, USA - November 11 - 16, 2012, page 60. ACM, 2012.

17. P. C. Nguy\&\#7877;n and D. Van Horn. Relatively complete counterexamples for higher-order programs. In Proceedings of the 36th ACM SIGPLAN Conference on Programming Language Design and Implementation, PLDI '15, pages 446-456, New York, NY, USA, 2015. ACM.

18. C. . L. Ong. On model-checking trees generated by higher-order recursion schemes. In 21st Annual IEEE Symposium on Logic in Computer Science (LICS'06), pages 81-90, Aug 2006.

19. A. M. Pitts. Nominal Sets: Names and Symmetry in Computer Science. Cambridge University Press, New York, NY, USA, 2013.

20. J. C. Reynolds. Definitional interpreters for higher-order programming languages. Higher-Order and Symbolic Computation, 11(4):363-397, 1998.

21. R. S. Boyer, B. Elspas, and K. Levitt. Select - a formal system for testing and debugging programs by symbolic execution. ACM SIGPLAN Notices, 10:234-245, 061975.

22. R. Sato, H. Unno, and N. Kobayashi. Towards a scalable software model checker for higher-order programs. In E. Albert and S. Mu, editors, Proceedings of the ACM SIGPLAN 2013 Workshop on Partial Evaluation and Program Manipulation, PEPM 2013, pages 53-62. ACM, 2013.

23. T. Terao and N. Kobayashi. A zdd-based efficient higher-order model checking algorithm. In J. Garrigue, editor, Programming Languages and Systems - 12th Asian Symposium, APLAS 2014, Singapore, November 17-19, 2014, Proceedings, volume 8858 of Lecture Notes in Computer Science, pages 354-371. Springer, 2014.

24. E. Torlak and R. Bodík. A lightweight symbolic virtual machine for solver-aided host languages. In M. F. P. O'Boyle and K. Pingali, editors, ACM SIGPLAN Conference on Programming Language Design and Implementation, PLDI '14, Edinburgh, United Kingdom - June 09 - 11, 2014, pages 530-541. ACM, 2014. 


\section{Appendix A Nominal determinacy}

While the operational semantics is bounded in depth, the reduction tree of a given term can still be infinite because of the non-determinacy involved in evaluating $\lambda$-abstractions: the rule non-deterministically creates a fresh name $m$ and extends the repository with $m$ mapped to the given $\lambda$-abstraction. This kind of nondeterminism, which can be seen as determinism up to fresh name creation, is formalised below.

Let us consider permutations $\pi$ : Meths $\rightarrow$ Meths such that, for all $m$, if $m \in$ Meths $_{\theta \rightarrow \theta^{\prime}}$ then $\pi(m) \in$ Meths $_{\theta \rightarrow \theta^{\prime}}$. We call such a permutation $\pi$ finite if the set $\{a \mid \pi(a) \neq a\}$ is finite. Given a syntactic object $X$ (e.g. a term, repository, or store) and a finite permutation $\pi$, we write $\pi \cdot X$ for the object we obtain from $X$ if we swap each name $a$ appearing in it with $\pi(a)$. Put otherwise, the operation $\cdot$ is an action from finite permutations of Meths to the set of objects $X$. Given a set $\Delta \subseteq$ Meths and objects $X, X^{\prime}$, we write $X \sim_{\Delta} X^{\prime}$ whenever there exists a finite permutation $\pi$ such that:

$$
\pi \cdot X=X^{\prime} \wedge \forall a \in \Delta . \pi(a)=a
$$

and say that $X$ and $X^{\prime}$ are nominally equivalent up to $\Delta$.

In the following lemma we write $\rightarrow_{n}$ for the $n$-step composition of $\rightarrow$.

Lemma 6 (Nominal determinacy). Let $(T, R, S, k)$ be a valid configuration, $(T, R, S, k) \rightarrow_{n}\left(T^{\prime}, R^{\prime}, S^{\prime}, k^{\prime}\right)$, and let $\Delta=\operatorname{dom}(R) \cup \operatorname{dom}(S)$. Then, for all $\left(T^{\prime \prime}, R^{\prime \prime}, S^{\prime \prime}, k^{\prime \prime}\right)$ we have $(T, R, S, k) \rightarrow_{n}\left(T^{\prime \prime}, R^{\prime \prime}, S^{\prime \prime}, k^{\prime \prime}\right)$ iff $\left(T^{\prime}, R^{\prime}, S^{\prime}, k^{\prime}\right) \sim_{\Delta}$ $\left(T^{\prime \prime}, R^{\prime \prime}, S^{\prime \prime}, k^{\prime \prime}\right)$.

\section{Appendix B Proof of Correctness Lemma}

Lemma 5 [Correctness] Given $M, R, C, D, \phi, \alpha, p c, k, \sigma$ such that $\sigma \simeq \phi$, $(M, R, D, k)\{\sigma\}$ is valid, and:

$$
\llbracket M, R, C, D, \phi, \alpha, p c, k \rrbracket=\left(\text { ret }, \phi^{\prime}, R^{\prime}, C^{\prime}, D^{\prime}, \alpha^{\prime}, p c^{\prime}\right)
$$

there exists $\sigma^{\prime} \supseteq \sigma$ such that $\sigma^{\prime} \simeq \phi^{\prime}$ and:

- if $(M, R, D, k)\{\sigma\} \rightarrow(v, \hat{R}, \hat{S}, \hat{k})$ then $\sigma^{\prime} \vDash\left(p c \Longrightarrow\left(p c^{\prime} \wedge(r e t=v)\right)\right) \wedge$ $\left((\right.$ inil $\left.\wedge \alpha \wedge p c) \Longrightarrow \alpha^{\prime}\right), R^{\prime}\left\{\sigma^{\prime}\right\} \supseteq \hat{R}$, and $D^{\prime}\left\{\sigma^{\prime}\right\}=\hat{S}$.

- if $(M, R, D, k)\{\sigma\} \rightarrow(E[\operatorname{assert}(0)], \hat{R}, \hat{S}, \hat{k})$ then $\sigma^{\prime} \vDash((\operatorname{inil} \wedge \alpha \wedge p c) \Longrightarrow$ $\left.\neg \alpha^{\prime}\right)$

- if $(M, R, D, k)\{\sigma\} \rightarrow(\hat{M}, \hat{R}, \hat{S}, \mathrm{nil})$ then $\sigma^{\prime} \vDash\left(p c \Longrightarrow \neg p c^{\prime}\right) \wedge(($ inil $\wedge \alpha \wedge$ $\left.p c) \Longrightarrow \alpha^{\prime}\right) \wedge\left((\neg\right.$ inil $\left.\wedge \alpha \wedge p c) \Longrightarrow \neg \alpha^{\prime}\right)$

where $\rightarrow$ is the reflexive transitive closure of $\rightarrow$.

Proof. Consider $\sigma \simeq \phi$, a valid configuration $(M, R, D, k)\{\sigma\}$, and its corresponding translation $\llbracket M, R, C, D, \phi, \alpha, p c, k \rrbracket=\left(\right.$ ret $\left., \phi^{\prime}, R^{\prime}, C^{\prime}, D^{\prime}, \alpha^{\prime}, p c^{\prime}\right)$.

Now, by induction on the length of the transition sequence produced by the operational semantics, and, lexicographically, by induction on the size of the term, we have the following cases for configurations in canonical form. 


\section{Terminal configurations:}

$-k=\mathrm{nil}$.

From the operational semantics we have $(M, R, D, \mathrm{nil})\{\sigma\}$ as a struck configuration. From the translation we have

$$
\begin{aligned}
& \llbracket M, R, C, D, \phi, \alpha, p c, k \rrbracket= \\
& \quad(\text { ret },(\text { ret }=\operatorname{defval}) \wedge \phi, R, C, D, \alpha \wedge(p c \Longrightarrow \text { inil }), \perp)
\end{aligned}
$$

We thus want show that $\exists \sigma^{\prime} \supseteq \sigma$ such that $\left(\sigma^{\prime} \simeq((\right.$ ret $\left.=\operatorname{defval}) \wedge \phi)\right)$ and

$$
\begin{aligned}
\sigma^{\prime} \vDash & (p c \Longrightarrow \neg \perp) \\
& \wedge((\text { inil } \wedge \alpha \wedge p c) \Longrightarrow(\alpha \wedge(p c \Longrightarrow \text { inil }))) \\
& \wedge((\neg \text { inil } \wedge \alpha \wedge p c) \Longrightarrow \neg(\alpha \wedge(p c \Longrightarrow \text { inil })))
\end{aligned}
$$

Let us choose $\sigma^{\prime}=\sigma[$ ret $\mapsto$ defval $]$. Since $\sigma \simeq \phi$, and since ret is the only new variable in $\phi^{\prime}$, we know that $\sigma^{\prime} \simeq \phi^{\prime}$. We also know that $\sigma^{\prime} \vDash(p c \Longrightarrow$ $\top) \wedge(($ inil $\wedge \alpha \wedge p c) \Longrightarrow(\alpha \wedge(p c \Longrightarrow$ inil $))) \wedge((\neg$ inil $\wedge \alpha \wedge p c) \Longrightarrow$ $\neg(\alpha \wedge(p c \Longrightarrow$ inil $)))$ holds.

$-M=\operatorname{assert}(0)$.

From the operational semantics we have (assert $(0), R, D, k)\{\sigma\}$ as a struck configuration. From the translation we have

$$
\begin{aligned}
& \llbracket \text { assert }(0), R, C, D, \phi, \alpha, p c, k \rrbracket= \\
& \quad(\text { ret },(\text { ret }=()) \wedge \phi, R, C, D, \alpha \wedge(p c \Longrightarrow(0 \neq 0)), \top)
\end{aligned}
$$

We thus want show that $\exists \sigma^{\prime} \supseteq \sigma$ such that $\left(\sigma^{\prime} \simeq((\right.$ ret $\left.=()) \wedge \phi)\right)$ and

$$
\sigma^{\prime} \vDash((\text { inil } \wedge \alpha \wedge p c) \Longrightarrow \neg(\alpha \wedge(p c \Longrightarrow(0 \neq 0))))
$$

Let us choose $\sigma^{\prime}=\sigma[$ ret $\mapsto()]$. Since $\sigma \simeq \phi$, and ret is the only new variable in $\phi^{\prime}$, we know that $\sigma^{\prime} \simeq \phi^{\prime}$. We also know that $\sigma^{\prime} \vDash(($ inil $\wedge \alpha \wedge p c) \Longrightarrow$ $\neg(\alpha \wedge(p c \Longrightarrow \perp)))$ holds.

$-M=v$.

From the operational semantics we have $(v, R, D, k)\{\sigma\}$ as a struck configuration. From the translation we have

$$
\begin{aligned}
& \llbracket v, R, C, D, \phi, \alpha, p c, k \rrbracket= \\
& \quad(\text { ret },(\text { ret }=v) \wedge \phi, R, C, D, \alpha, \top)
\end{aligned}
$$

We thus want show that $\exists \sigma^{\prime} \supseteq \sigma$ such that $\left(\sigma^{\prime} \simeq((\right.$ ret $\left.=v) \wedge \phi)\right)$ and

$$
\begin{aligned}
& \sigma^{\prime} \vDash(p c \Longrightarrow(\top \wedge(\text { ret }=v))) \wedge((\text { inil } \wedge \alpha \wedge p c) \Longrightarrow \alpha) \\
& R\left\{\sigma^{\prime}\right\} \supseteq R\left\{\sigma^{\prime}\right\} \text { and } D\left\{\sigma^{\prime}\right\}=D\left\{\sigma^{\prime}\right\}
\end{aligned}
$$

Let us choose $\sigma^{\prime}=\sigma[$ ret $\mapsto v]$. Since $\sigma \simeq \phi$, and ret is the only new variable in $\phi^{\prime}$, we know that $\sigma^{\prime} \simeq \phi^{\prime}$. We also know that $\sigma^{\prime} \vDash(p c \Longrightarrow(\top \wedge($ ret $=$ $v)) \wedge(($ inil $\wedge \alpha \wedge p c) \Longrightarrow \alpha)$ holds. Additionally, $R\left\{\sigma^{\prime}\right\} \supseteq R\left\{\sigma^{\prime}\right\}$ and $D\left\{\sigma^{\prime}\right\}=D\left\{\sigma^{\prime}\right\}$ hold trivially.

- $M=(v)$. Similar to the case above. 


\section{Non-terminal configurations:}

$-M=\operatorname{assert}(i)$ where $i \neq 0$.

From the operational semantics we have

$$
(\operatorname{assert}(i), R, D, k)\{\sigma\} \rightarrow((), R, D, k)\{\sigma\}
$$

From the translation we have

$$
\begin{aligned}
& \llbracket \operatorname{assert}(i), R, C, D, \phi, \alpha, p c, k \rrbracket= \\
& \quad(\operatorname{ret},(r e t=()) \wedge \phi, R, C, D, \alpha \wedge(p c \Longrightarrow(i \neq 0)), \top)
\end{aligned}
$$

We thus want show that $\exists \sigma^{\prime} \supseteq \sigma$ such that $\left(\sigma^{\prime} \simeq((\right.$ ret $\left.=()) \wedge \phi)\right)$ and, since this is a valid transition sequence, we want

$$
\begin{aligned}
& \sigma^{\prime} \vDash(p c \Longrightarrow(\text { ret }=())) \wedge((\text { inil } \wedge \alpha \wedge p c) \Longrightarrow(\alpha \wedge(p c \Longrightarrow(i \neq 0)))) \\
& R\left\{\sigma^{\prime}\right\} \supseteq R\left\{\sigma^{\prime}\right\} \text { and } D\left\{\sigma^{\prime}\right\}=D\left\{\sigma^{\prime}\right\}
\end{aligned}
$$

Let us choose $\sigma^{\prime}=\sigma[$ ret $\mapsto()]$. Since $\sigma \simeq \phi$, and ret is the only new variable in $\phi^{\prime}$, we know that $\sigma^{\prime} \simeq \phi^{\prime}$. We also know that $\sigma^{\prime} \vDash(p c \Longrightarrow($ ret $=()))$ holds because $\sigma^{\prime}$ maps ret to () , and $\sigma^{\prime} \vDash(($ inil $\wedge \alpha \wedge p c) \Longrightarrow \alpha)$ holds. Additionally, $R\left\{\sigma^{\prime}\right\} \supseteq R\left\{\sigma^{\prime}\right\}$ and $D\left\{\sigma^{\prime}\right\}=D\left\{\sigma^{\prime}\right\}$ hold trivially.

$-M=r:=v$.

From the operational semantics we have

$$
(r:=v, R, D, k)\{\sigma\} \rightarrow((), R, D[r \mapsto v], k)\{\sigma\}
$$

From the translation we have

$$
\begin{aligned}
& \llbracket r:=v, R, C, D, \phi, \alpha, p c, k \rrbracket= \\
& \quad \text { let } C^{\prime}=C[r] \text { in let } D\left[r \mapsto C^{\prime}(r)\right] \text { in } \\
& \quad\left(\text { ret },(r e t=()) \wedge\left(D^{\prime}(r)=v\right) \wedge \phi, R, C^{\prime}, D^{\prime}, \alpha, \top\right)
\end{aligned}
$$

We thus want show that $\exists \sigma^{\prime} \supseteq \sigma$ such that $\left(\sigma^{\prime} \simeq\left((\right.\right.$ ret $\left.\left.=()) \wedge\left(D^{\prime}(r)=v\right) \wedge \phi\right)\right)$ and, since this is a valid transition sequence, we want

$$
\begin{aligned}
& \sigma^{\prime} \vDash(p c \Longrightarrow(\text { ret }=())) \wedge((\text { inil } \wedge \alpha \wedge p c) \Longrightarrow(\alpha \wedge(p c \Longrightarrow(i \neq 0)))) \\
& R\left\{\sigma^{\prime}\right\} \supseteq R\left\{\sigma^{\prime}\right\} \text { and } D\left[r \mapsto C^{\prime}(r)\right]\left\{\sigma^{\prime}\right\}=D[r \mapsto v]\left\{\sigma^{\prime}\right\}
\end{aligned}
$$

Let us choose $\sigma^{\prime}=\sigma\left[\right.$ ret $\left.\mapsto(), C^{\prime}(r) \mapsto v\right]$. Since $\sigma \simeq \phi$, ret is the only new variable in $\phi^{\prime}$, and $D^{\prime}$ maps $r$ to $C^{\prime}(r)$, which $\sigma^{\prime}$ maps to $v$, we know that $\sigma^{\prime} \simeq \phi^{\prime}$. We also know that $\sigma^{\prime} \vDash(p c \Longrightarrow($ ret $=()))$ holds because $\sigma^{\prime}$ maps ret to () , and $\sigma^{\prime} \vDash(($ inil $\wedge \alpha \wedge p c) \Longrightarrow \alpha)$ holds. Additionally, $R\left\{\sigma^{\prime}\right\} \supseteq R\left\{\sigma^{\prime}\right\}$ holds trivially, and $D\left[r \mapsto C^{\prime}(r)\right]\left\{\sigma^{\prime}\right\}=D[r \mapsto v]\left\{\sigma^{\prime}\right\}$ holds because $\sigma^{\prime}$ maps $C^{\prime}(r)$ to $v$. 
$-M=! r$.

From the operational semantics we have

$$
(! r, R, D, k)\{\sigma\} \rightarrow(v, R\{\sigma\}, D\{\sigma\}, k) \quad \text { where } v=D\{\sigma\}(r)=\sigma(D(r))
$$

From the translation we have

$$
\begin{aligned}
& \llbracket ! r, R, C, D, \phi, \alpha, p c, k \rrbracket= \\
& \quad(\text { ret },(r e t=D(r)) \wedge \phi, R, C, D, \alpha, \top)
\end{aligned}
$$

We thus want show that $\exists \sigma^{\prime} \supseteq \sigma$ such that $\left(\sigma^{\prime} \simeq((\right.$ ret $\left.=D(r)) \wedge \phi)\right)$ and, since this is a valid transition sequence, we want

$$
\begin{aligned}
& \sigma^{\prime} \vDash(p c \Longrightarrow(\text { ret }=v)) \wedge((\text { inil } \wedge \alpha \wedge p c) \Longrightarrow \alpha) \\
& R\left\{\sigma^{\prime}\right\} \supseteq R\left\{\sigma^{\prime}\right\} \text { and } D\left\{\sigma^{\prime}\right\}=D\left\{\sigma^{\prime}\right\}
\end{aligned}
$$

Let us choose $\sigma^{\prime}=\sigma[$ ret $\mapsto D(r)]$. Since $\sigma \simeq \phi$, and ret is the only new variable in $\phi^{\prime}$, we know that $\sigma^{\prime} \simeq \phi^{\prime}$. We also know that $\sigma^{\prime} \vDash(p c \Longrightarrow($ ret $=v))$ holds because $\sigma^{\prime} \supset \sigma, \sigma(D(r))=v$ and $\sigma^{\prime}($ ret $)=D(r)$, and we know $\sigma^{\prime} \vDash(($ inil $\wedge \alpha \wedge p c) \Longrightarrow \alpha)$ holds. Additionally, $R\left\{\sigma^{\prime}\right\} \supseteq R\left\{\sigma^{\prime}\right\}$ and $D\left\{\sigma^{\prime}\right\}=D\left\{\sigma^{\prime}\right\}$ hold trivially.

- $M=v_{1} \oplus v_{2}$.

From the operational semantics we have

$$
\left(v_{1} \oplus v_{2}, R, D, k\right)\{\sigma\} \rightarrow(v, R\{\sigma\}, D\{\sigma\}, k) \quad \text { where } v=\sigma\left(v_{1}\right) \oplus \sigma\left(v_{2}\right)
$$

From the translation we have

$$
\begin{aligned}
& \llbracket v_{1} \oplus v_{2}, R, C, D, \phi, \alpha, p c, k \rrbracket= \\
& \quad\left(\text { ret },\left(\text { ret }=\left(v_{1} \oplus v_{2}\right)\right) \wedge \phi, R, C, D, \alpha, \top\right)
\end{aligned}
$$

We thus want show that $\exists \sigma^{\prime} \supseteq \sigma$ such that $\left(\sigma^{\prime} \simeq\left(\left(\right.\right.\right.$ ret $\left.\left.\left.=\left(v_{1} \oplus v_{2}\right)\right) \wedge \phi\right)\right)$ and, since this is a valid transition sequence, we want

$$
\begin{aligned}
& \sigma^{\prime} \vDash(p c \Longrightarrow(\text { ret }=v)) \wedge((\text { inil } \wedge \alpha \wedge p c) \Longrightarrow \alpha) \\
& R\left\{\sigma^{\prime}\right\} \supseteq R\left\{\sigma^{\prime}\right\} \text { and } D\left\{\sigma^{\prime}\right\}=D\left\{\sigma^{\prime}\right\}
\end{aligned}
$$

Let us choose $\sigma^{\prime}=\sigma[$ ret $\mapsto v]$. Since $\sigma \simeq \phi$, and ret is the only new variable in $\phi^{\prime}$, we know that $\sigma^{\prime} \simeq \phi^{\prime}$. We also know that $\sigma^{\prime} \vDash(p c \Longrightarrow($ ret $=v))$ holds because $\sigma^{\prime} \supseteq \sigma, \sigma\left(v_{1}\right) \oplus \sigma\left(v_{2}\right)=v$ and $\sigma^{\prime}$ maps ret to $v$. Lastly, we know $\sigma^{\prime} \vDash(($ inil $\wedge \alpha \wedge p c) \Longrightarrow \alpha)$ holds. Additionally, $R\left\{\sigma^{\prime}\right\} \supseteq R\left\{\sigma^{\prime}\right\}$ and $D\left\{\sigma^{\prime}\right\}=D\left\{\sigma^{\prime}\right\}$ hold trivially.

- $M=\pi_{i} v$.

From the operational semantics we have

$$
\left(\pi_{i} v, R, D, k\right)\{\sigma\} \rightarrow\left(v_{i}, R\{\sigma\}, D\{\sigma\}, k\right) \quad \text { where } \sigma(v)=\left\langle v_{1}, v_{2}\right\rangle
$$

From the translation we have

$$
\llbracket \pi_{i} v, R, C, D, \phi, \alpha, p c, k \rrbracket=
$$




$$
\left(\text { ret },\left(r e t=\left(\pi_{i} v\right)\right) \wedge \phi, R, C, D, \alpha, \top\right)
$$

We thus want show that $\exists \sigma^{\prime} \supseteq \sigma$ such that $\left(\sigma^{\prime} \simeq\left(\left(\right.\right.\right.$ ret $\left.\left.\left.=\left(\pi_{i} v\right)\right) \wedge \phi\right)\right)$ and, since this is a valid transition sequence, we want

$$
\begin{aligned}
& \sigma^{\prime} \vDash\left(p c \Longrightarrow\left(\text { ret }=v_{i}\right)\right) \wedge((\text { inil } \wedge \alpha \wedge p c) \Longrightarrow \alpha) \\
& R\left\{\sigma^{\prime}\right\} \supseteq R\left\{\sigma^{\prime}\right\} \text { and } D\left\{\sigma^{\prime}\right\}=D\left\{\sigma^{\prime}\right\}
\end{aligned}
$$

Let us choose $\sigma^{\prime}=\sigma\left[\right.$ ret $\left.\mapsto v_{i}\right]$. Since $\sigma \simeq \phi$, and ret is the only new variable in $\phi^{\prime}$, we know that $\sigma^{\prime} \simeq \phi^{\prime}$. We also know that $\sigma^{\prime} \vDash(p c \Longrightarrow($ ret $=v))$ holds because $\sigma^{\prime} \supseteq \sigma, \sigma\left(\pi_{i} v\right)=v_{i}$ and $\sigma^{\prime}$ maps ret to $v_{i}$. Lastly, we know $\sigma^{\prime} \vDash(($ inil $\wedge \alpha \wedge p c) \Longrightarrow \alpha)$ holds. Additionally, $R\left\{\sigma^{\prime}\right\} \supseteq R\left\{\sigma^{\prime}\right\}$ and $D\left\{\sigma^{\prime}\right\}=D\left\{\sigma^{\prime}\right\}$ hold trivially.

- $M=\lambda x . N$.

From the operational semantics we have

$$
(\lambda x . N, R, D, k)\{\sigma\} \rightarrow(\hat{m}, R[\hat{m} \mapsto \lambda x . N]\{\sigma\}, D\{\sigma\}, k)
$$

From the translation we have

$$
\begin{aligned}
& \llbracket \lambda x . N, R, C, D, \phi, \alpha, p c, k \rrbracket= \\
& \quad(\text { ret },(\text { ret }=m) \wedge \phi, R[m \mapsto \lambda x . N], C, D, \alpha, \top)
\end{aligned}
$$

We thus want show that $\exists \sigma^{\prime} \supseteq \sigma$ such that $\left(\sigma^{\prime} \simeq((\right.$ ret $\left.=m) \wedge \phi)\right)$ and, since this is a valid transition sequence, we want

$$
\begin{aligned}
& \sigma^{\prime} \vDash(p c \Longrightarrow(\text { ret }=\hat{m})) \wedge((\text { inil } \wedge \alpha \wedge p c) \Longrightarrow \alpha) \\
& R[m \mapsto \lambda x . N]\left\{\sigma^{\prime}\right\} \supseteq R[\hat{m} \mapsto \lambda x . N]\left\{\sigma^{\prime}\right\} \text { and } D\left\{\sigma^{\prime}\right\}=D\left\{\sigma^{\prime}\right\}
\end{aligned}
$$

Let us choose $\sigma^{\prime}=\sigma[$ ret $\mapsto m]$. Since $\sigma \simeq \phi$ and ret is the only new variable in $\phi^{\prime}$, and choosing $\hat{m}$ such that $\hat{m}=m$ by Lemma 6 (nominal determinism of the operational semantics), we know that $\sigma^{\prime} \simeq \phi^{\prime}$. We also know that $\sigma^{\prime} \vDash$ $(p c \Longrightarrow($ ret $=\hat{m}))$ holds because $\sigma^{\prime} \supset \sigma, m=\hat{m}$ by Lemma 6, and $\sigma^{\prime}$ maps ret to $m$. Lastly, we know $\sigma^{\prime} \vDash(($ inil $\wedge \alpha \wedge p c) \Longrightarrow \alpha)$ holds. Additionally, $D\left\{\sigma^{\prime}\right\}=D\left\{\sigma^{\prime}\right\}$ holds trivially, and $R[m \mapsto \lambda x . N]\left\{\sigma^{\prime}\right\} \supseteq R[\hat{m} \mapsto \lambda x . N]\left\{\sigma^{\prime}\right\}$.

- $M=m v$.

From the operational semantics we have

$$
(m v, R, S, k)\{\sigma\} \rightarrow(N\{v / x\}, R, S, k-1)\{\sigma\}
$$

where $R(m)=\lambda x . N$.

From the translation we have

$$
\llbracket m v, R, C, D, \phi, \alpha, p c, k \rrbracket=\llbracket N\{v / x\}, R, C, D, \phi, \alpha, p c, k-1 \rrbracket
$$

As such, this case holds directly from the inductive hypothesis. 
- $M=$ letrec $f=\lambda x . N$ in $M^{\prime}$.

From the operational semantics we have

(letrec $f=\lambda x . N$ in $\left.M^{\prime}, R, S, k\right)\{\sigma\} \rightarrow\left(M^{\prime}\{m / f\}, R[m \mapsto \lambda x . N], S, k\right)\{\sigma\}$

where $R(m)=\lambda x . N$.

From the translation we have

$$
\begin{aligned}
& \text { 【letrec } f=\lambda x . N \text { in } M^{\prime}, R, C, D, \phi, \alpha, p c, k \rrbracket= \\
& \quad \llbracket M\left\{f^{\prime} / f\right\}, R\left[m \mapsto \lambda x \cdot N\left\{f^{\prime} / f\right\}\right], C, D, \phi \wedge\left(f^{\prime}=m\right), \alpha, p c, k \rrbracket
\end{aligned}
$$

Let $\sigma^{\prime}=\sigma\left[f^{\prime} \mapsto m\right]$ such that $\sigma^{\prime} \simeq\left(\phi \wedge\left(f^{\prime}=m\right)\right)$. As such, this case holds directly from the inductive hypothesis.

- $M=\left(\right.$ let $x=v$ in $\left.M^{\prime}\right)$. Similar to the case above.

- $M=$ if $v$ then $M_{1}$ else $M_{0}$.

From the operational semantics we have

$$
\text { (if } \left.v \text { then } M_{1} \text { else } M_{0}, R, S, k\right)\{\sigma\} \rightarrow\left(M_{i}, R, S, k-1\right)\{\sigma\}
$$

where $i=0$ if $\sigma(v)=0$, and $i=1$ otherwise.

From the translation we have

【if $v$ then $M_{1}$ else $M_{0}, R, C, D, \phi, \alpha, p c, k \rrbracket=$

let $\left(\right.$ ret $\left._{0}, \phi_{0}, R_{0}, C_{0}, D_{0}, \alpha_{0}, p c_{0}\right)=\llbracket M_{0}, R, C, D, \phi, \alpha, p c \wedge(v=0), k \rrbracket$ in

let $\left(\right.$ ret $\left._{1}, \phi_{1}, R_{1}, C_{1}, D_{1}, \alpha_{1}, p c_{1}\right)=\llbracket M_{1}, R_{0}, C_{0}, D_{b}, \phi_{0}, \alpha_{0}, p c \wedge(v \neq 0), k \rrbracket$ in

let $C^{\prime}=C_{1}\left[r_{1}\right] \cdots\left[r_{n}\right]\left(\Pi=\left\{r_{1}, \ldots, r_{n}\right\}\right)$ in

let $\psi_{0}=(v=0) \Longrightarrow\left(\left(\right.\right.$ ret $\left.\left.=\operatorname{ret}_{0}\right) \wedge \bigwedge_{r \in \Pi}\left(C^{\prime}(r)=D_{0}(r)\right)\right)$ in

let $\psi_{1}=(v \neq 0) \Longrightarrow\left(\left(r e t=\operatorname{ret}_{1}\right) \wedge \bigwedge_{r \in \Pi}\left(C^{\prime}(r)=D_{1}(r)\right)\right)$ in

$\left(\right.$ ret $\left., \psi_{0} \wedge \psi_{1} \wedge \phi_{1}, R, C^{\prime}, C^{\prime}, \alpha_{1},\left(\left(p c_{0} \wedge(v=0)\right) \vee\left(p c_{1} \wedge(v \neq 0)\right)\right)\right)$

We now have two cases for $\sigma(v)$ :

1. $\sigma(v)=0$.

(1) By the inductive hypothesis we have $\sigma_{0} \supseteq \sigma$, where $\sigma_{0} \simeq \phi_{0}$, and all necessary conditions $P_{0}$ are satisfied.

(2) By Lemma 7, we know $\exists \sigma_{1} \supseteq \sigma_{0} \cdot\left(\sigma_{1} \simeq \phi_{1}\right)$.

Let $\sigma^{\prime}=\sigma_{1}\left[\right.$ ret $\mapsto$ ret $\left._{0}, C^{\prime}(r) \mapsto D_{0}(r)\right]$. Since $\sigma^{\prime} \supset \sigma_{1} \supseteq \sigma_{0}$, we know that $P_{0}$ is also satisfied. Thus, this case holds by (1) and (2).

2. $\sigma(v) \neq 0$.

(1) By Lemma 7, we know $\exists \sigma_{0} \supseteq \sigma$. $\left(\sigma_{0} \simeq \phi_{0}\right)$.

By Lemma 8, we know $R$ must be preserved in $R_{0}$, so $R_{0}\left\{\sigma_{0}\right\} \supseteq R$. Thus, by the inductive hypothesis:

(2) we have $\sigma_{1} \supseteq \sigma_{0} \supseteq \sigma$, where $\sigma_{1} \simeq \phi_{1}$, and all necessary conditions $P_{1}$ are satisfied.

Let $\sigma^{\prime}=\sigma_{1}\left[\right.$ ret $\left.\mapsto \operatorname{ret}_{1}, C^{\prime}(r) \mapsto D_{1}(r)\right]$. Since $\sigma^{\prime} \supset \sigma_{1}$, we know that $P_{1}$ is also satisfied. Thus, this case holds by (1) and (2). 
$-M=x v$.

From the operational semantics we have

$$
(x v, R, S, k)\{\sigma\} \rightarrow\left(N_{i}\left\{v / y_{i}\right\}, R, S, k-1\right)\{\sigma\}
$$

where $\sigma(x)=m_{i}$ and $R\left(m_{i}=\lambda y_{i} . N_{i}\right)$.

Let $\sigma_{0}=\sigma$.

From the translation we have

$$
\begin{aligned}
& \llbracket x^{\theta} v, R, C, D, \phi, \alpha, p c, k \rrbracket= \\
& \text { if } R \uparrow \theta=\emptyset \text { then }(\text { ret },(r e t=\mathrm{nil}) \wedge \phi, R, C, D, \alpha, p c) \text { else } \\
& \text { let } R\left\lceil\theta \text { be }\left\{m_{1}, \ldots, m_{n}\right\} \text { and }(R, C, \phi, \alpha) \text { be }\left(R_{0}, C_{0}, \phi_{0}, \alpha_{0}\right)\right. \text { in } \\
& \text { for each } i \in\{1, \ldots, n\}: \\
& \quad \text { let } R\left(m_{i}\right) \text { be } \lambda y_{i} . N \text { in } \\
& \quad \text { let }\left(r e t_{i}, \phi_{i}, R_{i}, C_{i}, D_{i}, \alpha_{i}, p c_{i}\right)= \\
& \quad \llbracket N_{i}\left\{v / y_{i}\right\}, R_{i-1}, C_{i-1}, D, \phi_{i-1}, \alpha_{i-1}, p c \wedge\left(x=m_{i}\right), k-1 \rrbracket \text { in } \\
& \text { let } C_{n}^{\prime}=C_{n}\left[r_{1}\right] \cdots\left[r_{j}\right]\left(\Pi=\left\{r_{1}, \ldots, r_{j}\right\}\right) \text { in } \\
& \text { let } \psi=\bigwedge_{i=1}^{n}\left(\begin{array}{l}
\left(x=m_{i}\right) \Longrightarrow \\
\left(\left(r e t=r e t_{i}\right) \wedge\right. \\
\left.\bigwedge_{r \in \Pi}\left(C_{n}^{\prime}(r)=D_{i}(r)\right)\right)
\end{array}\right) \text { in } \\
& \text { let } p c_{n}^{\prime}=\bigvee_{i=1}^{n}\left(p c_{i} \wedge\left(x=m_{i}\right)\right) \text { in } \\
& \left(\text { ret } \psi \wedge \phi_{n}, R_{n}, C_{n}^{\prime}, C_{n}^{\prime}, \alpha_{n}, p c_{n}^{\prime}\right)
\end{aligned}
$$

Since $R\left\lceil\theta=\left\{m_{1}, \ldots, m_{n}\right\}\right.$, it must be the case that $i \in\{1 \ldots n\}$.

It must be the case then that either (1) $i=1$ or (2) $1<i \leq n$.

1. (1) By the inductive hypothesis, we have $\exists \sigma_{1} \supseteq \sigma_{0} \cdot\left(\sigma_{1} \simeq \phi_{1}\right)$, and all necessary properties $P_{0}$ hold.

(2) By Lemma 7 applied repeatedly, we have $\exists \sigma_{n} \supseteq \cdots \supseteq \sigma_{1} \cdot\left(\sigma_{n} \simeq \phi_{n}\right)$. Since $\sigma_{n} \supseteq \sigma_{1}$, properties $P_{0}$ hold, so this case holds by (1) and (2).

2. (1) By Lemma 7 applied repeatedly, we know $\exists \sigma_{i-1} \supseteq \cdots \supseteq \sigma_{0} \cdot\left(\sigma_{i-1} \simeq\right.$ $\left.\phi_{i-1}\right) \wedge \cdots \wedge\left(\sigma_{0} \simeq \phi_{0}\right)$.

(2) By Lemma 8 applied repeatedly, we also know $R_{i-1} \supseteq \cdots \supseteq R_{0}$.

(3) By the inductive hypothesis, we know $\exists \sigma_{i} \supseteq \sigma_{i-1} .\left(\sigma_{i} \simeq \phi_{i}\right)$ and the necessary properties $P_{i}$ hold.

(4) By Lemma 7 again applied repeatedly, we know $\exists \sigma_{n} \supseteq \cdots \supseteq \sigma_{i} \cdot\left(\sigma_{n} \simeq\right.$ $\left.\phi_{n}\right) \wedge \cdots \wedge\left(\sigma_{i} \simeq \phi_{i}\right)$.

Since $\sigma_{n} \supseteq \sigma_{i}$, properties $P_{i}$ hold, so this holds by (1), (2), (3) and (4). - $M=\left(\right.$ let $x=M^{\prime}$ in $\left.M^{\prime \prime}\right)$, with $M^{\prime}$ not a value.

Let us write $M$ as $E\left[M^{\prime}\right]$. From the operational semantics we have

$$
\left(E\left[M^{\prime}\right], R, S, k\right)\{\sigma\} \rightarrow(E[\hat{M}], \hat{R}, \hat{S}, \hat{k})\{\sigma\} \rightarrow \ldots
$$


where $\left(M^{\prime}, R, S, k\right)\{\sigma\} \rightarrow(\hat{M}, \hat{R}, \hat{S}, \hat{k})\{\sigma\}$.

We now have the following translation.

$$
\begin{aligned}
& \llbracket E\left[M^{\prime}\right], R, C, D, \phi, \alpha, p c, k \rrbracket= \\
& \quad \text { let }\left(\text { ret }_{1}, \phi_{1}, R_{1}, C_{1}, D_{1}, \alpha_{1}, p c_{1}\right)=\llbracket M^{\prime}, R, C, D, \phi, \alpha, p c, k \rrbracket \text { in } \\
& \quad \text { let }\left(\text { ret }_{2}, \phi_{2}, R_{2}, C_{2}, D_{2}, \alpha_{2}, p c_{2}\right)=\llbracket E\left[\text { ret }_{1}\right], R_{1}, C_{1}, D_{1}, \phi_{1}, \alpha_{1}, p c \wedge p c_{1}, k \rrbracket \text { in } \\
& \quad\left(\text { ret }_{2}, \phi_{2}, R_{2}, C_{2}, D_{2}, \alpha_{2}, p c_{1} \wedge p c_{2}\right)
\end{aligned}
$$

Since $E\left[M^{\prime}\right]$ can lead to either (1) some value, (2) an assertion assert(0), or (3) a stuck configuration where the bound is nil, we have three cases to consider.

1. $\left(E\left[M^{\prime}\right], R, S, k\right)\{\sigma\} \rightarrow(E[\hat{M}], \hat{R}, \hat{S}, \hat{k})\{\sigma\} \rightarrow\left(E\left[\hat{v_{1}}\right], \hat{R_{1}}, \hat{S_{1}}, \hat{k_{1}}\right)$.

(1) By the inductive hypothesis: $\sigma_{1} \vDash\left(p c \Longrightarrow\left(p c_{1} \wedge\left(\right.\right.\right.$ ret $\left.\left.\left._{1}=\hat{v_{1}}\right)\right)\right) \wedge$ $\left((p c \wedge \alpha \wedge\right.$ inil $\left.) \Longrightarrow \alpha_{1}\right)$ and $R_{1}\left\{\sigma_{1}\right\} \supseteq R, D_{1}\left\{\sigma_{1}\right\}=\hat{S}$.

$\left(E\left[\hat{v}_{1}\right], \hat{R}_{1}, \hat{S}_{1}, \hat{k_{1}}\right) \rightarrow\left(\hat{v}^{\prime}, \hat{R}^{\prime}, \hat{S}^{\prime}, \hat{k}\right)^{\prime}$.

(2) By the inductive hypothesis: $\sigma_{2} \vDash\left(p c_{1} \Longrightarrow\left(p c_{2} \wedge\left(\right.\right.\right.$ ret $\left.\left.\left._{2}=\hat{v}^{\prime}\right)\right)\right) \wedge$ $\left(\left(p c_{1} \wedge \alpha_{1} \wedge\right.\right.$ inil $\left.) \Longrightarrow \alpha_{2}\right)$ and $R_{2}\left\{\sigma_{2}\right\} \supseteq \hat{R}_{1}, D\left\{\sigma_{1}\right\}=\hat{S}$.

Since $\sigma_{2} \supseteq \sigma$, we know $\sigma_{2} \simeq \phi_{2}$.

From (1) and (2), we know $\sigma_{2} \vDash\left(p c \Longrightarrow\left(p c_{1} \wedge p c_{2} \wedge(\right.\right.$ ret $\left.\left.=\hat{v})\right)\right) \wedge((p c \wedge$ $\alpha \wedge$ inil $\left.) \Longrightarrow \alpha_{2}\right)$. Case holds.

2. (a) $\left(E\left[M^{\prime}\right], R, S, k\right)\{\sigma\} \rightarrow(E[\hat{M}], \hat{R}, \hat{S}, \hat{k})\{\sigma\} \rightarrow\left(E[\operatorname{assert}(0)], \hat{R_{1}}, \hat{S_{1}}, \hat{k_{1}}\right)$.

(1) By the inductive hypothesis: $\sigma_{1} \vDash\left((p c \wedge \alpha \wedge\right.$ inil $\left.) \Longrightarrow \neg \alpha_{1}\right)$ such that $\sigma_{1} \simeq \phi_{1}$.

(2) By Lemma 7, we know $\exists \sigma_{2} \supseteq \sigma_{1} . \sigma_{2} \simeq \phi_{2}$

By Lemma 9 and (1) and (2), we know $\alpha_{2} \Longrightarrow \alpha_{1}$, thus $\neg \alpha_{1} \Longrightarrow$ $\neg \alpha_{2}$. We therefore have $\sigma_{1} \vDash\left((p c \wedge \alpha \wedge\right.$ inil $\left.) \Longrightarrow \neg \alpha_{2}\right)$. Case holds.

(b) $\left(E\left[M^{\prime}\right], R, S, k\right)\{\sigma\} \rightarrow(E[\hat{M}], \hat{R}, \hat{S}, \hat{k})\{\sigma\} \rightarrow\left(E\left[\hat{v_{1}}\right], \hat{R_{1}}, \hat{S_{1}}, \hat{k_{1}}\right)$.

(1) By the inductive hypothesis: $\sigma_{1} \vDash\left(p c \Longrightarrow\left(p c_{1} \wedge\left(\right.\right.\right.$ ret $_{1}=$ $\left.\left.\left.\hat{v_{1}}\right)\right)\right) \wedge\left((p c \wedge \alpha \wedge\right.$ inil $\left.) \Longrightarrow \alpha_{1}\right)$ and $R_{1}\left\{\sigma_{1}\right\} \supseteq R, D_{1}\left\{\sigma_{1}\right\}=\hat{S}$.

$\left(E\left[\hat{v}_{1}\right], \hat{R}_{1}, \hat{S_{1}}, \hat{k_{1}}\right) \rightarrow\left(E^{\prime}[\operatorname{assert}(0)], \hat{R}^{\prime}, \hat{S}^{\prime}, \hat{k}\right)^{\prime}$

(2) By the inductive hypothesis: $\sigma_{2} \vDash\left(\left(p c \wedge p c_{1} \wedge \alpha_{1} \wedge\right.\right.$ inil $\left.) \Longrightarrow \neg \alpha_{2}\right)$ such that $\sigma_{2} \simeq \phi_{2}$.

From (1) and (2) we have $\sigma_{2} \vDash\left(p c \Longrightarrow \neg\left(p c_{1} \wedge p c_{2}\right)\right) \wedge(\alpha \wedge$ $p c \wedge$ inil $) \Longrightarrow \neg \alpha_{2}$. Case holds.

3. (a) $\left(E\left[M^{\prime}\right], R, S, k\right)\{\sigma\} \rightarrow(E[\hat{M}], \hat{R}, \hat{S}, \hat{k})\{\sigma\} \rightarrow\left(E\left[\hat{M}_{1}\right], \hat{R}_{1}, \hat{S}_{1}\right.$, nil $)$.

(1) By the inductive hypothesis: $\sigma_{1} \vDash\left(p c \Longrightarrow \neg p c_{1}\right) \wedge(($ inil $\wedge \alpha \wedge$ $\left.p c) \Longrightarrow \alpha_{1}\right) \wedge\left((\neg\right.$ inil $\left.\wedge \alpha \wedge p c) \Longrightarrow \neg \alpha_{1}\right)$ such that $\sigma_{1} \simeq \phi_{1}$.

(2) By Lemma 7, we know $\exists \sigma_{2} \supseteq \sigma_{1} . \sigma_{2} \simeq \phi_{2}$.

(3) By Lemma 9 , we know $\alpha_{2} \Longrightarrow \alpha_{1}$, so $\neg \alpha_{1} \Longrightarrow \neg \alpha_{2}$.

From (1) we have that $\sigma_{1} \vDash\left(p c \Longrightarrow \neg\left(p c_{1} \wedge p c_{2}\right)\right)$. 
From (1) and (3) we have that $\sigma_{1} \vDash\left((\right.$ inil $\left.\wedge \alpha \wedge p c) \Longrightarrow \alpha_{2}\right) \wedge$ $\left((\neg\right.$ inil $\left.\wedge \alpha \wedge p c) \Longrightarrow \neg \alpha_{2}\right)$.

From (2) we have that $\sigma_{2} \vDash\left(p c \Longrightarrow \neg\left(p c_{1} \wedge p c_{2}\right)\right) \wedge(($ inil $\wedge \alpha \wedge$ $\left.p c) \Longrightarrow \alpha_{2}\right) \wedge\left((\neg\right.$ inil $\left.\wedge \alpha \wedge p c) \Longrightarrow \neg \alpha_{2}\right)$ such that $\sigma_{2} \simeq \phi_{2}$. Case holds.

(b) $\left(E\left[M^{\prime}\right], R, S, k\right)\{\sigma\} \rightarrow(E[\hat{M}], \hat{R}, \hat{S}, \hat{k})\{\sigma\} \rightarrow\left(E\left[\hat{v}_{1}\right], \hat{R_{1}}, \hat{S_{1}}, \hat{k_{1}}\right)$.

(1) By the inductive hypothesis: $\sigma_{1} \vDash\left(p c \Longrightarrow\left(p c_{1} \wedge\left(\right.\right.\right.$ ret $_{1}=$ $\left.\left.\hat{v_{1}}\right)\right) \wedge\left((p c \wedge \alpha \wedge\right.$ inil $\left.) \Longrightarrow \alpha_{1}\right)$ and $R_{1}\left\{\sigma_{1}\right\} \supseteq R, D_{1}\left\{\sigma_{1}\right\}=\hat{S}$.

$\left(E\left[\hat{v}_{1}\right], \hat{R_{1}}, \hat{S_{1}}, \hat{k_{1}}\right) \rightarrow\left(E^{\prime}\left[\hat{M}^{\prime}\right], \hat{R}^{\prime}, \hat{S}^{\prime}, \mathrm{nil}\right)^{\prime}$

(2) By the inductive hypothesis: $\sigma_{2} \vDash\left(\left(p c \wedge p c_{1}\right) \Longrightarrow \neg p c_{2}\right) \wedge(($ inil $\wedge$ $\left.\left.\alpha_{1} \wedge p c \wedge p c_{1}\right) \Longrightarrow \alpha_{2}\right) \wedge\left(\left(\neg\right.\right.$ inil $\left.\left.\wedge \alpha_{1} \wedge p c \wedge p c_{1}\right) \Longrightarrow \neg \alpha_{2}\right)$ such that $\sigma_{2} \simeq \phi_{2}$.

From (1) and (2) we have $\sigma_{2} \vDash\left(p c \Longrightarrow \neg\left(p c_{1} \wedge p c_{2}\right)\right) \wedge(($ inil $\wedge \alpha \wedge$ $\left.p c) \Longrightarrow \alpha_{2}\right) \wedge\left((\neg\right.$ inil $\left.\wedge \alpha \wedge p c) \Longrightarrow \neg \alpha_{2}\right)$ such that $\sigma_{2} \simeq \phi_{2}$. Case holds.

Lemma 7 (Uniqueness of the translation). Given an assignment $\sigma$ and formula $\phi$ such that $\sigma \cong \phi$, and a translation

$$
\llbracket M, R, C, D, \phi, \alpha, p c, k \rrbracket=\left(\text { ret }, \phi^{\prime}, R^{\prime}, C^{\prime}, D^{\prime}, \alpha^{\prime}, p c^{\prime}\right)
$$

we know there exists some $\sigma^{\prime} \supseteq \sigma$ such that $\sigma^{\prime} \cong \phi^{\prime}$.

Proof. Assuming $\sigma \cong \phi$, by induction on $k$ and then by structural induction on $M$, we have the base cases:

1. $k=$ nil: shown by choosing $\sigma^{\prime}=\sigma[$ ret $\mapsto$ defval $]$.

2. $M=\operatorname{assert}(() v)$ and $k \neq$ nil: shown by choosing $\sigma^{\prime}=\sigma[$ ret $\mapsto()]$.

3. $M=v$ and $k \neq$ nil: shown by choosing $\sigma^{\prime}=\sigma[$ ret $\mapsto v]$.

4. $M=! r$ and $k \neq$ nil: shown by choosing $\sigma^{\prime}=\sigma[r e t \mapsto D(r)]$.

5. $M=\lambda x . N$ and $k \neq$ nil: shown by choosing $\sigma^{\prime}=\sigma[$ ret $\mapsto m]$.

6. $M=\pi_{i} v$ and $k \neq$ nil: shown by choosing $\sigma^{\prime}=\sigma\left[\right.$ ret $\left.\mapsto \pi_{i} v\right]$.

7. $M=v_{1} \oplus v_{2}$ and $k \neq$ nil: shown by choosing $\sigma^{\prime}=\sigma\left[\right.$ ret $\left.\mapsto v_{1} \oplus v_{2}\right]$.

8. $M=r:=v$ and $k \neq$ nil: shown by choosing $\sigma^{\prime}=\sigma\left[\right.$ ret $\left.\mapsto(), D^{\prime}(r)=v\right]$.

With base cases done, we have the following inductive cases:

1. $M=$ let $x=M^{\prime}$ in $M^{\prime \prime}$ :

(1) By the inductive hypothesis on $\llbracket M, R, C, D, \phi, \alpha, p c, k \rrbracket$, we have $\sigma_{1} \simeq \phi_{1}$.

(2) By the inductive hypothesis on $\llbracket M^{\prime}\left\{\operatorname{ret}_{1} / x\right\}, R_{1}, C_{1}, D_{1}, \phi_{1}, \alpha_{1}, p c \wedge p c_{1}, k \rrbracket$, we have $\sigma_{2} \simeq \phi_{2}$.

This case holds by (1) and (2).

2. $M=$ letrec $f=\lambda x . N$ in $M^{\prime}$ :

Let $\sigma^{\prime}=\sigma\left[f^{\prime} \mapsto m\right]$ such that $\sigma^{\prime} \simeq\left(\phi \wedge\left(f^{\prime}=m\right)\right)$.

(1) By the inductive hypothesis on $\llbracket M^{\prime}\left\{f^{\prime} / f\right\}, R^{\prime}, C, D, \phi \wedge\left(f^{\prime}=m\right), \alpha, p c, k \rrbracket$, we have $\sigma_{1} \simeq \phi_{1}$.

This case holds by (1). 
3. $M=m v$ :

(1) By the inductive hypothesis on $\llbracket N\{v / x\}, R^{\prime}, C, D, \phi, \alpha, p c, k \rrbracket$, we have $\sigma_{1} \simeq \phi_{1}$.

This case holds by (1).

4. $M=$ if $v$ then $M_{1}$ else $M_{0}$ :

(1) By the inductive hypothesis on $\llbracket M_{0}, R, C, D, \phi, \alpha, p c \wedge(v=0), k \rrbracket$, we have $\sigma_{0} \simeq \phi_{0}$.

(2) By the inductive hypothesis on $\llbracket M_{1}, R_{0}, C_{0}, D_{0}, \phi_{0}, \alpha_{0}, p c \wedge(v \neq 0), k \rrbracket$, we have $\sigma_{1} \simeq \phi_{1}$.

We now have two cases on $\sigma_{1}(v)$ :

(a) $\sigma_{1}(v)=0$. Choose $\sigma^{\prime}=\sigma_{1}\left[\right.$ ret $\left.\mapsto \operatorname{ret}_{0}, C^{\prime}(r) \mapsto D_{0}(r)\right]$ for all $r \in \Pi$.

(b) $\sigma_{1}(v)=i \neq 0$. Choose $\sigma^{\prime}=\sigma_{1}\left[\right.$ ret $\left.\mapsto \operatorname{ret}_{1}, C^{\prime}(r) \mapsto D_{1}(r)\right]$ for all $r \in \Pi$. 5. $M=x v$ :

(1) By the inductive hypothesis on $\llbracket N_{1}, R_{0}, C_{0}, \ldots \rrbracket$ to $\llbracket N_{n}, R_{n-1}, C_{n-1}, \ldots \rrbracket$, we have $\exists \sigma_{n} \supseteq \cdots \supseteq \sigma_{1} \supseteq \sigma_{0} \cdot\left(\sigma_{n} \simeq \phi_{n}\right) \wedge \cdots \wedge\left(\sigma_{1} \simeq \phi_{1}\right) \wedge\left(\sigma_{0} \simeq \phi_{0}\right)$.

Since $\sigma(x) \in R$, let $\sigma(x)=m_{i}$ for $i \in\{1 . . n\}$.

Let $\sigma^{\prime}=\sigma_{n}\left[\right.$ ret $\mapsto$ ret $\left._{i}, C_{n}^{\prime}(r) \mapsto D_{i}(r)\right]$ for all $r \in \Pi$.

This case holds by (1).

Lemma 8 (Preservation of the repository). Given a translation

$$
\llbracket M, R, C, D, \phi, \alpha, p c, k \rrbracket=\left(\text { ret }, \phi^{\prime}, R^{\prime}, C^{\prime}, D^{\prime}, \alpha^{\prime}, p c^{\prime}\right)
$$

we know the input repository must be preserved; i.e. $R^{\prime} \supseteq R$.

Proof. By inspection of the translation rules.

Lemma 9 (Propagation of preconditions). Given a translation

$$
\llbracket M, R, C, D, \phi, \alpha, p c, k \rrbracket=\left(\text { ret }, \phi^{\prime}, R^{\prime}, C^{\prime}, D^{\prime}, \alpha^{\prime}, p c^{\prime}\right)
$$

we know that preconditions $\phi$ and $\alpha$ must be propagated and included in $\phi^{\prime}$ and $\alpha^{\prime}$; i.e. $\phi^{\prime}=\psi \wedge \phi$ and $\alpha^{\prime}=\beta \wedge \alpha$ where $\llbracket M, R, C, D, \top, \top, \top, k \rrbracket=$ $\left(r e t, \psi, R^{\prime}, C^{\prime}, D^{\prime}, \beta, p c^{\prime \prime}\right)$

Proof. By inspection of the translation rules. 


\section{Appendix C Time Taken (s) for BMC-2 and MoCHi}

\begin{tabular}{|c|c|c|c|c|c|c|c|c|c|c|c|c|c|c|c|c|}
\hline & 1 & 2 & 3 & 4 & 5 & 6 & 7 & 8 & 9 & 10 & 11 & 12 & 13 & 14 & 15 & IoCHi \\
\hline $100 \_1-e$ & 018 & 0.018 & 0.028 & 0.034 & 0.029 & 0.056 & 0.173 & 0.312 & 0.605 & 1.661 & 5.640 & 2.778 & 84.130 & - & - & c \\
\hline $100 \_2$ & 013 & 0.017 & 0.017 & 0.020 & 0.018 & 0.029 & 0.028 & 0.032 & 0.036 & 0.032 & 0.050 & 0.053 & 0.053 & 0.062 & 0.071 & $\mathrm{c}$ \\
\hline $100 \_3-e$ & 013 & 0.016 & 0.015 & 0.021 & 0.024 & 0.018 & 0.027 & 0.022 & 0.025 & 0.028 & 0.034 & 0.035 & 0.040 & 0.056 & 0.051 & 10.734 \\
\hline 100_4-e & 012 & 0.019 & 0.017 & 0.022 & 0.026 & 0.025 & 0.026 & 0.037 & 0.034 & 0.036 & 0.037 & 0.047 & 0.040 & .046 & 0.063 & 13.547 \\
\hline $100 \_5-e$ & 0.007 & 0.015 & 0.019 & 0.025 & 0.028 & 0.048 & 0.168 & 0.344 & 0.576 & 1.520 & 5.911 & 18.821 & 90.544 & - & 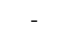 & 2.345 \\
\hline $200 \_1-e$ & 0.016 & 0.020 & 0.021 & 0.034 & 0.054 & 0.074 & 0.188 & 0.348 & 0.660 & 1.572 & 4.635 & 20.209 & 71.296 & - & - & $\mathrm{m}$ \\
\hline $200 \_2-e$ & 0.012 & 0.023 & 0.019 & 0.033 & 0.042 & 0.063 & 0.063 & 0.094 & 0.106 & 0.151 & 0.175 & 0.201 & 0.259 & 0.298 & 0.372 & - \\
\hline $200 \_3-e$ & 015 & 0.020 & 0.030 & 0.034 & 0.110 & 0.453 & 2.849 & 19.257 & - & - & - & - & - & - & $\mathrm{m}$ & 1.742 \\
\hline 0 _4-e & .030 & 0.034 & 0.047 & 0.051 & 0.102 & 0.401 & 2.661 & 18.285 & - & - & - & - & - & - & $\mathrm{m}$ & 47.022 \\
\hline $200 \_5-e$ & 0.043 & 0.036 & 0.037 & 0.040 & 0.047 & 0.046 & 0.065 & 0.085 & .111 & .146 & 0.168 & .218 & .282 & 0.388 & 0.428 & $\mathrm{~m}$ \\
\hline $400 \_1-e$ & 0.019 & 0.034 & 0.060 & 0.108 & 0.232 & 0.814 & 3.805 & 24.594 & - & - & - & - & - & - & $\mathrm{m}$ & $\mathrm{m}$ \\
\hline $400 \_2-e$ & 0.036 & 0.057 & 0.050 & 0.061 & 0.080 & 0.129 & 0.196 & 0.350 & 0.523 & 0.696 & 0.789 & 1.033 & .321 & .594 & 1.991 & - \\
\hline ack & 0.011 & 0.011 & 0.018 & 0.027 & 0.138 & 1.403 & 11.519 & 168.670 & - & - & - & - & - & - & - & 0.525 \\
\hline -cppr & .033 & 0.021 & 0.028 & 0.031 & 0.018 & 0.020 & 0.020 & 0.018 & 0.018 & 0.026 & 0.026 & 0.023 & .027 & 0.026 & 0.028 & 28.584 \\
\hline a-init-me & 0.018 & 0.023 & 0.031 & 0.027 & 0.027 & 0.024 & 0.015 & 0.017 & 0.021 & 0.025 & 0.029 & 0.029 & .034 & 0.040 & 0 & $\mathrm{c}$ \\
\hline a-init & 0.014 & 0.011 & 0.013 & 0.018 & 0.021 & 0.015 & 0.016 & 0.021 & 0.018 & 0.032 & 0.026 & 0.040 & .042 & 0.053 & 0.053 & c \\
\hline$a-m a x-m e$ & 0.008 & 0.013 & 0.020 & 0.023 & 0.017 & 0.021 & 0.018 & 0.016 & 0.016 & 0.017 & 0.015 & 0.019 & 0.019 & 0.018 & 0.022 & 5.348 \\
\hline a-max & 0.012 & 0.011 & 0.010 & 0.014 & 0.015 & 0.017 & 0 & 0 & 0.015 & 0.014 & 0 & 16 & 18 & 017 & 0 & .636 \\
\hline copy_i & .016 & 0.019 & 0.016 & 0.016 & 19 & 0.012 & 0.020 & 20 & 0.021 & 022 & 0.020 & 23 & 20 & 024 & 0.017 & 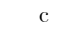 \\
\hline e-fact & 0.010 & 0.008 & 0.008 & 0.009 & 0.013 & 0.011 & 0.014 & 0.014 & 0.015 & 0.016 & 0.015 & 0.016 & .021 & 0.020 & 0.022 & 0.629 \\
\hline e-simple & 0.010 & 0.010 & 0.013 & 0.010 & 0.011 & 0.011 & 0.008 & 0.012 & 0.012 & 0.007 & 0.012 & 0.012 & 0.009 & 0.010 & 0.009 & 0.098 \\
\hline hors & 0.010 & 0.007 & 0.010 & 0.010 & 0.013 & 0.013 & 0.009 & 0.012 & 0.012 & 0.013 & 0.012 & 0.011 & 0.012 & 0.012 & 0.014 & 0.321 \\
\hline hrec & 0.012 & 0.015 & 0.020 & 0.020 & 0.024 & 0.028 & 0.075 & 0.362 & 3.284 & 26.175 & - & - & 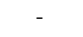 & - & - & 0.867 \\
\hline$t$ & 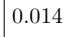 & 0 & 0 & 0.013 & 0 & 0 & & & 0 & & & & & .007 & & .123 \\
\hline intro3 & 0.011 & 0.013 & 0.015 & 0.011 & 0.012 & 0.011 & 0.013 & 0.009 & 0.010 & 0.009 & 0.011 & 0.010 & .008 & 0.012 & 0.007 & 0.110 \\
\hline 1-zipmap & 0.009 & 0.012 & 0.015 & 0.016 & 0.012 & 0.018 & 0.021 & 0.019 & 0.026 & 0.026 & 0.026 & 0.023 & 0.031 & 0.031 & 0.033 & c \\
\hline l-zipunzip & 0.013 & 0.011 & 0.008 & 0.009 & 0.016 & 0.017 & 0.019 & 0.022 & 0.014 & 0.025 & 0.030 & 0.037 & 0.048 & 0.049 & 0.062 & c \\
\hline $\max$ & 0.012 & 0.009 & 0.011 & 0.008 & 0.011 & 0.009 & 0.013 & 0.011 & 0.011 & 0.013 & 0.013 & 0.011 & 0.011 & 0.013 & 0.013 & 0.172 \\
\hline mc91-e & 0.010 & 0.013 & 0.010 & 0.012 & 0.017 & 0.052 & 0177 & 0230 & 0.895 & 3.513 & 12.128 & 71.43 & - & - & - & 5.119 \\
\hline mc91 & 012 & 0. & 0.013 & 0.018 & 0 & 0 & 0 & 0.348 & 0 & 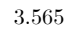 & 2.137 & 73.717 & 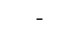 & - & - & .173 \\
\hline mult-e-m2 & 0.009 & 0.012 & 0.012 & 0.011 & 0.012 & 0.012 & 0.013 & 0.019 & 0.018 & 0.015 & 0.019 & 0.021 & 0.022 & 0.024 & 0.021 & 0.426 \\
\hline mult-e-m3 & 0.012 & 0.014 & 0.014 & 0.012 & 0.012 & 0.016 & 0.018 & 0.014 & 0.018 & 0.018 & 0.014 & 0.012 & 0.018 & 0.018 & 0.017 & 0.152 \\
\hline mult-e & 0.015 & 0.012 & 0.015 & 0.018 & 0.012 & 0.016 & 0.018 & 0.016 & 0.018 & 0.017 & 0.021 & 0.021 & 0.017 & 0.017 & 0.016 & $\mathrm{c}$ \\
\hline mult & 0.013 & 0.015 & 0.016 & 0.016 & 0.016 & 0.016 & 0.015 & 0.017 & 0.016 & 0.01 & 0.014 & 0.01 & & 0.014 & 0.011 & 0.153 \\
\hline repeat-e & 0.011 & 0.013 & 0.013 & 0.014 & 0.012 & 0.013 & 0.013 & 0.011 & 0.013 & 0.011 & 0.016 & 0.012 & & 0.014 & 0.012 & 0.189 \\
\hline r-lock-e & 0.009 & 0.009 & 0.013 & 0.009 & 0.013 & 0.011 & 0.013 & 0.012 & 0.013 & 0.013 & 0.009 & 0.010 & 97 & 0.011 & 0.011 & 0.216 \\
\hline r-lock & 0.007 & 0.012 & 0.009 & 0.009 & 0.010 & 0.013 & 0.013 & 0.010 & 0.011 & 0.010 & 0.010 & 0.011 & 0.012 & 0.012 & 0.006 & 0.135 \\
\hline sum-e & 0.008 & 0.013 & 0.009 & 0.013 & 0.012 & 0.014 & 0.015 & 0.013 & 0.017 & 0.015 & 0.010 & 0.014 & 0.015 & 0.016 & 0.013 & 0.145 \\
\hline sum-mult-e & 0.007 & 0.011 & 0.013 & 0.016 & 0.019 & 0.022 & 0.050 & 0.146 & 0.218 & 0.207 & 0.237 & 0.286 & 0.323 & 0.328 & 0.301 & 0.238 \\
\hline sum & 0.011 & 0.010 & 0.013 & 0.013 & 0.013 & 0.014 & 0.012 & 0.012 & 0.011 & 0.013 & 0.013 & 0.016 & 0.016 & 0.015 & 0.017 & 0.330 \\
\hline ref-1 & 0.007 & 0.009 & 0.014 & 0.013 & 0.011 & 0.007 & 0.007 & 0.011 & 0.012 & 0.013 & 0.010 & 0.011 & 0.012 & 0.011 & 0.011 & $\mathrm{u}$ \\
\hline ref-1-e & 0.023 & 0.007 & 0.019 & 0.016 & 0.020 & 0.020 & 0.008 & 0.010 & 0.013 & 0.014 & 0.013 & 0.014 & 0.014 & 0.012 & 0.009 & $\mathrm{u}$ \\
\hline ref-2 & 0.011 & 0.007 & 0.011 & 0.013 & 0.009 & 0.010 & 0. & 0.011 & 0.012 & 0.011 & 0.010 & 0.010 & 0.012 & 0.009 & 0.010 & $\mathrm{u}$ \\
\hline $\mathrm{re}$ & 0.011 & 0.008 & 0.009 & 0.011 & 0.009 & 0.012 & 0.013 & 0.013 & 0.013 & 0.010 & 0.008 & 0.011 & 0.013 & 0.011 & 0.011 & $\mathrm{u}$ \\
\hline ref-3 & 0.012 & 0.013 & 0.015 & 0.019 & 0.016 & 0.015 & 0.018 & 0.022 & 0.028 & 0.047 & 0.099 & 0.209 & 0.211 & 0.211 & 0.211 & $\mathrm{u}$ \\
\hline
\end{tabular}

